OPEN ACCESS

Check for updates

\title{
Effectiveness of physical activity monitors in adults: systematic review and meta-analysis
}

\author{
Rasmus Tolstrup Larsen, ${ }^{1,2}$ Vibeke Wagner, ${ }^{3}$ Christoffer Bruun Korfitsen, ${ }^{4,5}$ Camilla Keller, ${ }^{2,6}$ \\ Carsten Bogh Juhl, ${ }^{7,8}$ Henning Langberg, ${ }^{9}$ Jan Christensen ${ }^{2}$
}

For numbered affiliations see end of the article

Correspondence to: R T Larsen rala@sund.ku.dk

(or @RTolstrupLarsen on Twitter ORCID 0000-0002-8860-4233) Additional material is published online only. To view please visit the journal online.

Cite this as: BMJ 2022;376:e068047 http://dx.doi.org/10.1136/ bmj-2021-068047

Accepted: 29 November 2021

\section{ABSTRACT}

OBJECTIVE

To estimate the effectiveness of physical activity monitor (PAM) based interventions among adults and explore reasons for the heterogeneity.

\section{DESIGN}

Systematic review and meta-analysis.

\section{STUDY SELECTION}

The electronic databases MEDLINE, Embase, SPORTDiscus, CINAHL, and the Cochrane Central Register of Controlled Trials (CENTRAL) were searched on 4 June 2021. Eligible randomised controlled trials compared interventions in which adults received feedback from PAMs with control interventions in which no feedback was provided. No restrictions on type of outcome measurement, publication date, or language were applied.

\section{DATA EXTRACTION AND SYNTHESIS}

Two reviewers independently extracted data and assessed risk of bias. Random effects metaanalyses were used to synthesise the results. The certainty of evidence was rated by the Grading of Recommendations Assessment and Evaluation (GRADE) approach.

\section{MAIN OUTCOME MEASURES}

The three primary outcomes of interest were physical activity, moderate to vigorous physical activity, and sedentary time.

RESULTS

121 randomised controlled trials with 141 study comparisons, including 16743 participants, were included. The PAM based interventions showed a

\section{WHAT IS ALREADY KNOWN ON THIS TOPIC}

Modern physical activity monitors have the potential to be used as facilitators for behavioural change, providing direct feedback on activity to the user

In 2007 a systematic review reported that use of physical activity monitors could increase physical activity, but few studies were included and the effect estimate was affected by imprecision

Since 2007 several studies have been published with different conclusions about the effectiveness of physical activity monitors

\section{WHAT THIS STUDY ADDS}

This comprehensive and methodologically sound systematic review and metaanalysis summarises the existing body of evidence covering 121 studies, including 16743 participants

This study provides evidence for using physical activity monitors for enhancing physical activity and moderate to vigorous physical activity

Large scale studies and studies investigating sedentary time in general, especially among overweight participants, are needed to clarify evidence gaps identified here moderate effect (standardised mean difference 0.42, $95 \%$ confidence interval 0.28 to 0.55 ) on physical activity, equivalent to 1235 daily steps; a small effect $(0.23,0.16$ to 0.30$)$ on moderate to vigorous physical activity, equivalent to 48.5 weekly minutes; and a small insignificant effect $(-0.12,-0.25$ to 0.01$)$ on sedentary time, equal to 9.9 daily minutes. All outcomes favoured the PAM interventions.

\section{CONCLUSIONS}

The certainty of evidence was low for the effect of PAM based interventions on physical activity and moderate for moderate to vigorous physical activity and sedentary time. PAM based interventions are safe and effectively increase physical activity and moderate to vigorous physical activity. The effect on physical activity and moderate to vigorous physical activity is well established but might be overestimated owing to publication bias.

STUDY REGISTRATION

PROSPERO CRD42018102719.

\section{Introduction}

Physical inactivity, an activity level insufficient to meet current recommendations, has a large impact on global public health, as it is one of the major risk factors for non-communicable diseases and is estimated to be responsible for $9 \%$ of all premature deaths globally. ${ }^{1}$ Physical activity, any bodily movement produced by skeletal muscles that requires energy expenditure, ${ }^{2}$ has been quantified with physical activity monitors (PAMs) for research purposes for decades. ${ }^{3}$ However, as well as tracking and measuring physical activity, modern PAMs hold the potential to be used as facilitators for behavioural change, providing direct feedback on physical activity to the user. ${ }^{4}$ A novel systematic review by Bravata and colleagues in 2007 reported that PAMs could be effectively used to increase physical activity levels among adults. ${ }^{5}$ However, the number of randomised controlled trials included was low, and the effect estimate was affected by imprecision. Furthermore, several randomised controlled trials have been published since 2007 with different conclusions about the effectiveness of the PAMs. Some trials have reported promising effect sizes, ${ }^{6-12}$ some have reported inconclusive results owing to a lack of power or intervention effects, ${ }^{13-19}$ and some have reported negative findings on intervention effects. ${ }^{20-24}$ Even though several systematic reviews have investigated the effectiveness of PAMs, they have all been focused on specific populations or specific types of PAM or have included a limited number of randomised controlled trials. $^{525-46}$ On the basis of the published literature, PAMs are expected to be effectively increasing physical 
activity behaviour in general. Nevertheless, as no systematic review has included all available studies, the evidence on the effectiveness of PAM based interventions promoting physical activity among all adults needs to be systematically reviewed according to best practice recommendations from the Cochrane Collaboration to provide high quality guidelines for a diverse audience with an interest in general medicine and public health. ${ }^{47}$

The objective of this systematic review and metaanalysis was to estimate the effect on physical activity, moderate and vigorous physical activity, and sedentary time from PAM based interventions compared with control interventions in which the participants did not receive feedback from PAMs in participants aged 18-65 years. Subsequently, we examined whether the effectiveness was affected by characteristics of studies and participants.

\section{Methods}

This systematic review and meta-analysis was conducted according to the recommendations from Cochrane and is reported according to the Preferred Reporting Items for Systematic Reviews and MetaAnalyses (PRISMA) statement. ${ }^{47} 48$ The study protocol holds a detailed description of the methods, has been registered in the PROSPERO database, and has previously been published. ${ }^{49}$

\section{Eligibility criteria}

We included randomised controlled trials and randomised crossover trials. For studies to be considered eligible for inclusion, more than $80 \%$ of the study participants had to be above 18 years of age and below 65 years of age. We calculated age distributions by following the methods used by Hall and colleagues. ${ }^{50}$ We included studies comparing any PAM based intervention in which the participants received feedback on their physical activity level from the PAMs. The PAMs may be portable or wearable, electronic or mechanical, and driven by accelerometers, pedometers, or global positioning systems. In all control interventions, the participants could not receive any feedback on their physical activity level from the PAMs

\section{Outcomes}

The three primary outcomes of interest were changes in physical activity, moderate to vigorous physical activity, and sedentary time. If more than one relevant outcome was reported in a study on physical activity, we favoured daily step counts followed by daily metres walked and daily energy expenditure. Finally, if no objective measure was available for physical activity, we used self-reported measures. If more than one relevant outcome was reported in a study on moderate to vigorous physical activity, we favoured objectively measured activity followed by self-reported activity. If more than one relevant outcome was reported in a study on sedentary time, we favoured objectively measured sedentary time followed by self-reported sedentary time. For all three primary outcomes, we used the individual study definitions of physical activity, moderate to vigorous physical activity, and sedentary time. If a study reported only either physical activity or moderate to vigorous physical activity, we included the study results in the specific analysis only for that outcome. We extracted reported adverse events and dropouts.

\section{Search methods for identification of studies}

We searched the electronic databases MEDLINE, Embase, SPORTDiscus, CINAHL, and the Cochrane Central Register of Controlled Trials (CENTRAL) on 4 June 2021. The search string combined relevant keywords and MeSH/thesaurus terms for PAMs and study design. The search matrix is reported in the study protocol along with the full search strings. ${ }^{49}$ We contacted the authors of all unobtainable studies or studies with missing data. We applied no restrictions on language or publication date. Two reviewers (CK and VW) independently used citation pearl growing to hand search references of eligible studies and reviews identified from the search. We searched the database ClinicalTrials.gov to identify ongoing trials. We contacted the study authors of trial protocols if the trial status was uncertain (for example, if the anticipated completion date was overdue but no published study could be identified).

\section{Study selection, data extraction, and risk of bias}

A combination of two reviewers (RTL, VW, CBK, CK, JC) independently screened all titles and abstracts of identified studies. At least one of the reviewers assessed the full text of articles judged to be eligible, and consensus on eligibility was reached by discussion. Two reviewers (RTL, VW, CBK, CK, CBJ, JC) independently extracted data and assessed risk of bias using the RoB 2.0 tool. $^{51}$ Disagreements between reviewers were solved by consulting a third reviewer. To provide the most realistic comparisons, we used the most active control interventions as comparators if more than one control group was available in a study (for example, other non-PAM based behavioural change interventions over usual care interventions over wait list). We imputed median values as means and extracted the standard deviation; estimated it from the standard error, 95\% confidence interval, $\mathrm{P}$ value, or interquartile range; or measured it on a graph, as recommended by the Cochrane Handbook. ${ }^{52}$ Studies without any quantification of variance for endpoint scores had standard deviations imputed from baseline measures.

\section{Data synthesis}

As described in a previous study protocol, ${ }^{49}$ we calculated the effect size as a standardised mean difference of the final scores and summarised it using a Hartung-Knapp-Sidik-Jonkman random effects meta-analysis after adjustment to Hedges' g. ${ }^{53} 54$ We estimated the risk ratio for adverse events and dropouts, and studies with zero events were given 0.5 events for both the intervention and control group to 
be included. ${ }^{55}$ We extracted no follow-up data after the end of intervention scores.

We tested the heterogeneity of results by using the Cochrane $\mathrm{Q}$ test and quantified it as $\mathrm{I}^{2}$ values and the between study variance $\tau^{2}$. We assessed small study bias by calculating the Egger's test score; if this was significant, we used a trim and fill method to adjust for small study bias by removing the studies that caused the small study bias and then imputing missing studies on the bias corrected estimate. ${ }^{5657}$ Furthermore, we used a non-parametric Spearman's rank correlation test to investigate the relation between standardised mean difference and standard error; as a non-protocol defined sensitivity analysis, we used a Copas selection model to investigate the possibility of unpublished trials. ${ }^{58}$ For all statistical analyses, we considered an $\alpha$ level of 0.05 to be statistically significant. We used RStudio version 1.3.1093, using R version 4.0.3, for all analyses and illustrations.

\section{Subgroup analyses and analysis of heterogeneity}

We investigated heterogeneity by doing subgroup analyses and stratified analyses on the following nominal variables: diagnoses, feedback frequency, and content of control intervention (active versus nonactive control). The description of these analyses can be found in the study protocol. However, we did not do the protocol defined analyses on type of intervention other than feedback from PAMs (for example, different types of behavioural change intervention or medical interventions related to the specific patient population of individual studies) owing to the complexity of the prevailing classification. We did not do the protocol defined subgroup analyses on whether the participants received feedback on their disease risks according to their physical activity level owing to insufficient data. Two reviewers (JC and RTL) independently rated the certainty of evidence for each outcome by using the Grading of Recommendations Assessment and Evaluation (GRADE) approach (domains used to assess the certainty of the evidence were risk of bias, inconsistency, indirectness, imprecision, and publication bias). ${ }^{59} 60$ We used the Cochrane rule of thumb $(<0.4$ interpreted as a small effect, 0.4-0.7 interpreted as a moderate effect, $>0.7$ interpreted as a large effect) to re-express the standardised mean differences in the summary of findings. ${ }^{61}$ As a sensitivity approach, we did cumulative meta-analyses to investigate by which year the pooled estimates were positive with at least a clinically relevant small effect (standardised mean difference $<0.2$ with $95 \%$ confidence). ${ }^{62}$ Another deviation from the study protocol was the use of gross national income per capita as a measure of a country's economy to explain the heterogeneity of the study results, over the protocol defined use of a country's gross domestic product. We did this because the World Bank uses gross national income to classify low, lower middle, upper middle, and high income countries. ${ }^{63}$

Some studies used non-blinded or non-sealed PAMs to assess the study groups during baseline and endpoint weeks. To investigate whether the pooled effect estimates were affected directly by bias due to deviations from the intended control interventions, we did a non-protocol defined sensitivity analysis to analyse whether the control groups were considered to be exposed for feedback from the PAMs.

\section{Patient and public involvement}

Because this review did not focus on any specific patient population, no patients were directly involved in setting the research question or the outcome measures or in the design or implementation of the study. No patients were asked to advise on interpretation or writing up of results. However, the members of the research team have worked with physical activity behaviour among different patient populations, which have inspired this review. Patient representatives will be included in the dissemination of results, including the use of lay summaries describing the research and its results for non-scientific audiences.

\section{Results}

In total, we included 121 studies with 141 study comparisons and 16743 participants. ${ }^{6-12}$ 14-24 64-167 The search was conducted on 4 June 2021 and identified 18253 unique study references. Hand searching of 59 relevant reviews identified five additional studies. ${ }^{717593106110}$ We contacted 105 study authors for obtaining of full text or protocol details, confirmation of trial status, or sharing of preliminary or missing relevant data. The 67 unobtainable studies excluded as "no full text" were conference abstracts, inaccessible trials, or completed trial registrations for which the study report could not be found and the authors did not respond. Figure 1 shows the study selection process and reasons for exclusion in the full text screening.

\section{Characteristics of included studies}

All 121 included studies were conducted in high income countries except for eight studies that were conducted in upper middle income countries. ${ }^{128087104123129150156}$ Most of the included studies were European (31\%) or North American (40\%). Most of the studies were categorised as including mainly healthy participants (47\%), followed by studies that reported including overweight participants (17\%) and studies that reported including participants with cancer (12\%). In total, $62 \%$ of the included studies used passive control comparisons, meaning that most of the control groups' participants received no active intervention content; $81 \%$ of the included studies used goal setting for the intervention group participants; and almost all (97\%) of the included studies provided daily feedback on physical activity for the intervention group participants. The median duration of intervention among the included studies was 12 weeks. The median baseline daily step count was 6994 , and the median body mass index was 27.8. The median age of the participants was 47 years, and the median proportion of female participants was 


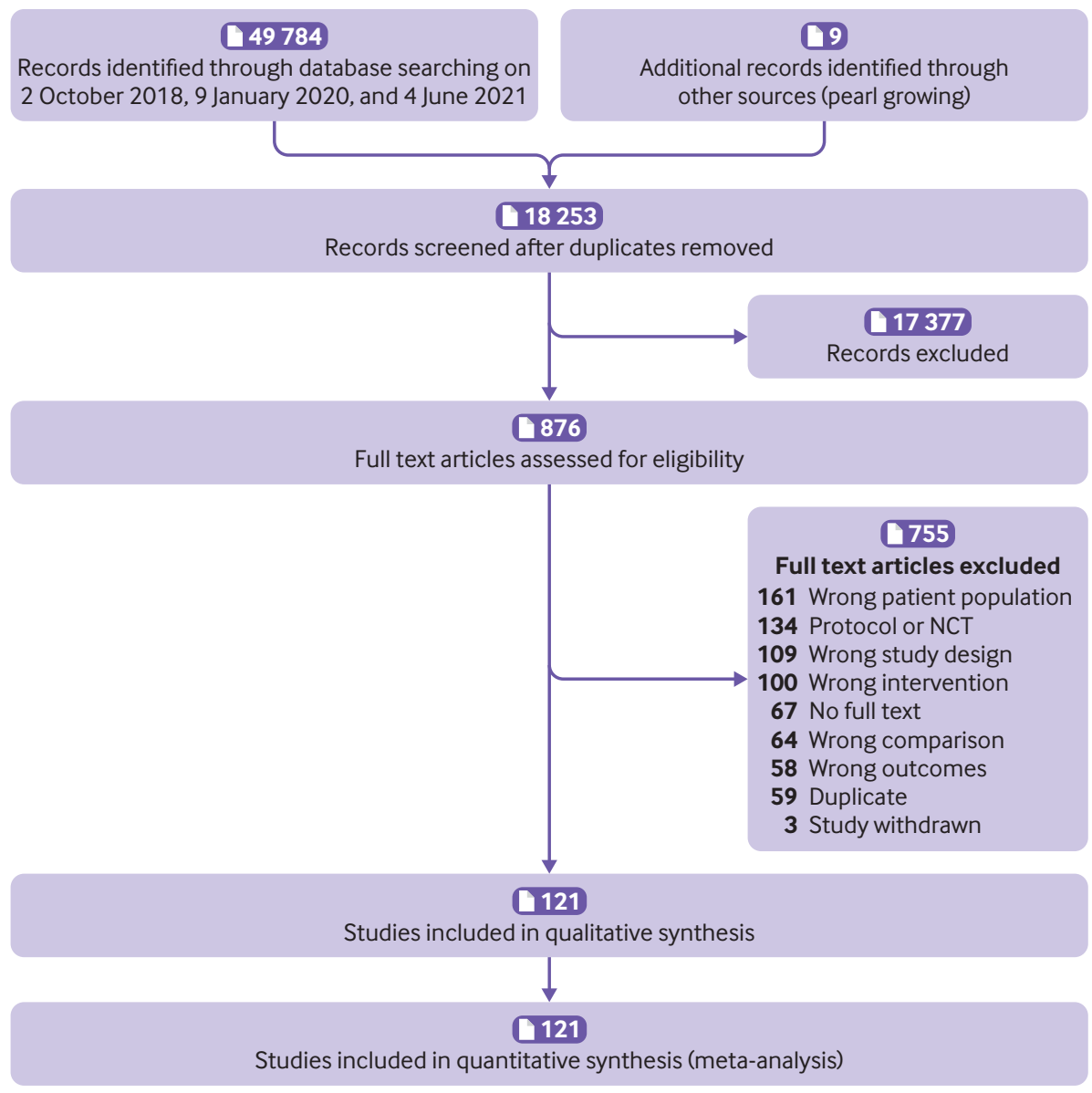

Fig 1 | PRISMA flow diagram illustrating selection of studies. NCT=National Clinical Trial registry

$77 \%$. The median sample size of the included studies was 69 . Table 1 summarises the characteristics of the included studies, with detailed summary statistics and references. Appendix 1 provides information about interventions, types of PAM used in the intervention and for measuring outcomes, and further study level characteristics.

\section{Risk of bias in individual studies}

Risk of bias assessments in individual studies, including reasons, are listed in the characteristics of included studies in appendix 1. Figure 2 illustrates the risk of bias for the three outcomes.

\section{Effects of interventions on physical activity}

We included 103 studies including results for 12840 participants in the meta-analysis on physical activity. 6-12 14-16 18-24 64-69 73-80 82 83 85-94 96-101 103 104 106-126 128-137 139-141 143 145-148 150-154 156158159 161-164 167 The

overall standardised mean difference was $0.42(95 \%$ confidence interval 0.28 to $0.55 ; \mathrm{I}^{2}=81 \%$ ) in favour of the PAM interventions. When we transformed the standardised mean difference to a weighted mean difference on daily steps using a median standard deviation of 2940 steps, ${ }^{145}$ the pooled effect equated to 1235 (95\% confidence interval 823 to 1617 ) daily steps in favour of the intervention. No methodological heterogeneity (whether active or passive control interventions were used; whether objective or selfreported outcome instruments were used; whether goal setting was applied; whether the participants received feedback daily, weekly, or monthly; how long the interventions lasted; what gross national income the study country had; or how the studies were assessed in terms of risk of bias) or clinical heterogeneity (participant population, baseline step count, age of participants, sex distribution of participants, or body mass index of participants) explained the heterogeneity of the results significantly or relevantly. Because of funnel plot asymmetry (positive Eggers' test, intercept 2.03 (95\% confidence interval 1.32 to 2.75)) and, therefore, a risk of small study bias, we applied a trim and fill method in which 36 fictive studies were added, giving an adjusted standardised mean difference of 0.15 ( -0.02 to 0.32). A Copas selection model suggested that 69 studies were left unpublished and gave an adjusted standardised mean difference of 0.15 (0.06 to 0.24 ). A cumulative metaanalysis showed that the standardised mean difference has been significantly larger than 0.25 since 2014 . Two comparisons had standard deviations imputed for daily steps, ${ }^{110} 165$ as did one comparison for weekly walking minutes. ${ }^{91}$ Three comparisons reported only change scores and were included in the analysis after calculation of the end of treatment standardised mean difference. ${ }^{16}{ }^{125}$ Three studies reported results 

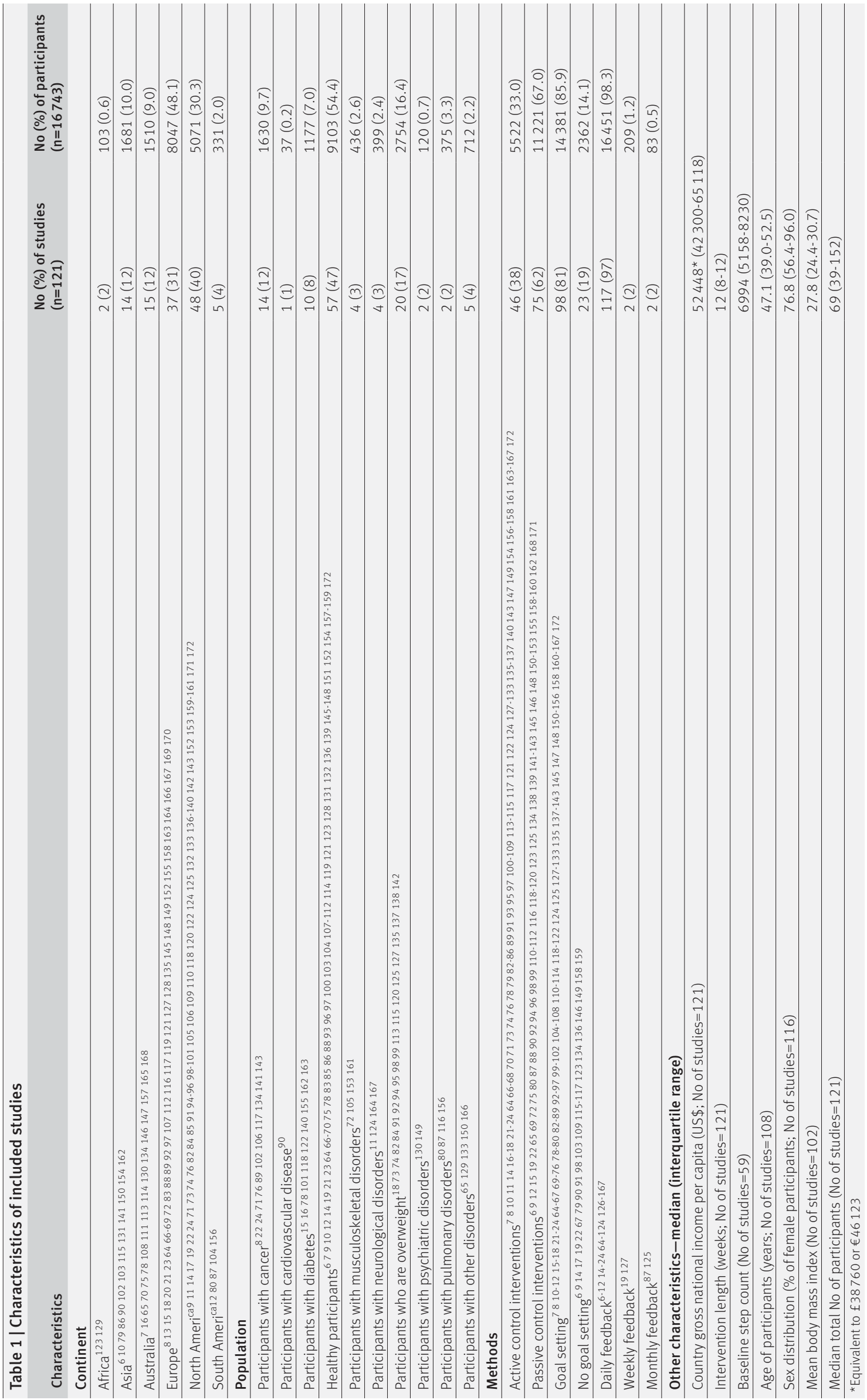

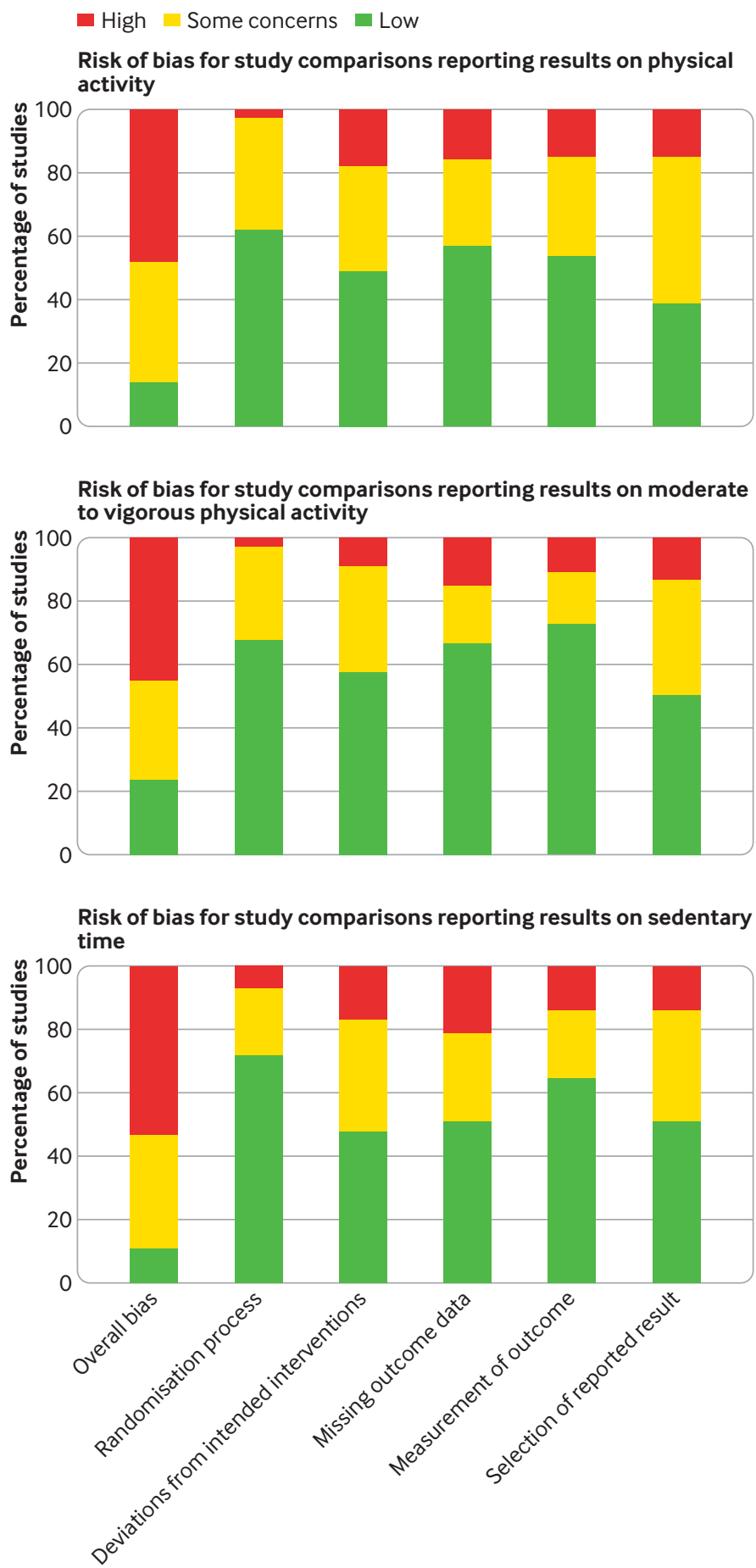

Fig 2 | Risk of bias for three outcomes

from dichotomous outcomes and were included in the analysis after calculation of the odds ratio and estimation of the individual standardised mean difference. $^{1889101}$

\section{Effects of interventions on moderate to vigorous physical activity}

We included 63 studies including results on 8250 participants in the meta-analysis on moderate to vigorous physical activity. ${ }^{711} 12152022-246769-7476$ 82-87 90 92-95 97-100 $102105106108111 \quad 114-117119124$ 126-128 132134
138142143145146149153155157 162-164166167172 The overall standardised mean difference was 0.23 (0.16 to 0.30 ; $\mathrm{I}^{2}=67 \%$ ) in favour of the PAM interventions. When we transformed the standardised mean difference to a weighted mean difference on weekly minutes of moderate to vigorous physical activity using a median standard deviation of 211 minutes, ${ }^{165}$ the pooled effect equated to 48.5 (33.8 to 63.3) minutes of moderate to vigorous physical activity in favour of the intervention. The following covariates and risk of bias items explained some of the heterogeneity of the results. Studies using objective outcome instruments for moderate to vigorous physical activity reported a lower standardised mean difference $(0.14,0.06$ to 0.22$)$ than did studies using self-reported measures (0.42, 0.22 to 0.62). Studies with low risk of bias arising from the randomisation process were found to have a larger standardised mean difference $(0.28,0.16$ to 0.40$)$ than studies with some concerns $(0.04,0.00$ to 0.08$)$ and those with high risk of bias $(0.17,-3.10$ to 3.44$)$. Studies with high risk of bias in selection of the reported results were found to have a lower standardised mean difference $(0.05$, -0.04 to 0.14$)$ than studies with some concerns $(0.31$, 0.20 to 0.42$)$ and those with low risk of bias $(0.24$, 0.09 to 0.38 ). No other methodological heterogeneity (whether active or passive control interventions were used; whether goal setting was applied; whether the participants received feedback daily, weekly, or monthly; how long the interventions lasted; what gross national income the study country had; or how the studies were assessed in terms of other risk of bias domains) or clinical heterogeneity (participant population, baseline step count, age of participants, sex distribution of participants, or body mass index of participants) explained the heterogeneity of the results significantly or relevantly. Because of funnel plot asymmetry (positive Eggers' test, intercept 1.00 (0.59 to 1.42)) and the consequent risk of small study bias, we applied a trim and fill method in which 22 fictive studies were added, giving an adjusted standardised mean difference of 0.06 ( -0.01 to 0.13$)$. A Copas selection model suggested that 21 studies were left unpublished and gave an adjusted standardised mean difference of 0.10 (0.03 to 0.17). A cumulative metaanalysis showed that the standardised mean difference has been significantly larger than 0.15 since 2016 . One comparison had standard deviations imputed for weekly moderate to vigorous physical activity. ${ }^{67}$ Two comparisons reported only change scores and were included in the analysis after calculation of the end of treatment standardised mean difference. ${ }^{111142}$ One study reported results from dichotomous outcomes and was included in the analysis after calculation of the odds ratio and estimation of the individual standardised mean difference. ${ }^{84}$

\section{Effects of interventions on sedentary time}

We included 38 studies including results on 5634 participants in the meta-analysis on sedentary time. ${ }^{13}$ $22236466677073828792-949899103105113114116117119121$ 126-128130143146149152153155157 160-163 172 The overall 
standardised mean difference was $-0.12(-0.25$ to $0.01 ; \mathrm{I}^{2}=66 \%$ ), favouring the PAM intervention as the intervention groups were less sedentary. When we transformed the standardised mean difference to a weighted mean difference of daily sedentary minutes using a median standard deviation of 87.2 minutes, the pooled effect equated to 9.9 ( -0.8 to 21.8 ) daily minutes of sedentary time less in the intervention groups. Participant population groups were found to explain some heterogeneity $(\mathrm{P}<0.001)$, primarily driven by the results from studies with overweight participants having an effect size favouring the control interventions $(0.11,-0.02$ to 0.23$)$ compared with the other participant populations. Country's gross national income was correlated with the effect of the interventions (coefficient $0.01,95 \%$ confidence interval 0.00 to 0.02 ) per US $\$ 10000$ ( $€ 7390$; €8794) increase). No other methodological heterogeneity (whether active or passive control interventions were used; whether objective or self-reported outcome instruments were used; whether goal setting was applied; whether the participants received feedback daily, weekly, or monthly; how long the interventions lasted; or how the studies were assessed in terms of risk of bias) or clinical heterogeneity (baseline step count, age of participants, sex distribution of participants, or body mass index of participants) explained the heterogeneity of the results significantly or relevantly. No funnel plot asymmetry, and therefore no risk of small study bias, was found. A cumulative meta-analysis showed that the pooled estimate has been stable around a small effect since 2020. One comparison had standard deviations imputed for weekly sitting minutes. ${ }^{67}$

Appendix 2 shows the forest plots from the random effects meta-analyses. Appendix 3 gives the full results from the subgroup analyses and meta-regressions.

\section{Adverse events and discontinued interventions}

With 224 (6.4\%) adverse events among 3501 intervention group participants and 186 (5.5\%) adverse events among 3355 control group participants, we found no significant association between group allocation when summarising the risk of experiencing an adverse event in 34 studies (relative risk 1.1, 95\% confidence interval 0.93 to $1.30 ; \mathrm{I}^{2}=0 \%$ ). With 931 $(10.1 \%)$ of 9201 intervention group participants discontinuing the interventions and 713 (8.5\%) among 8374 control group participants discontinuing the interventions, we found no significant association between group allocation when summarising the risk of discontinuing interventions in 117 studies (relative risk 1.1, 0.96 to $1.20 ; \mathrm{I}^{2}=16 \%$ ).

\section{Non-protocol defined sensitivity analyses on} whether control group participants were considered to receive feedback from PAMs

We found no effect from whether the control group participants were considered to be exposed for nonblinded PAMs in baseline and endpoint measurement weeks for physical activity and moderate to vigorous physical activity. For sedentary time, the studies in which the control group was considered to receive feedback had a less favourable effect size $(0.06,-0.62$ to 0.74$)$ than the other studies $(-0.14,-0.27$ to -0.00$)$.

\section{Summary of findings and risk of bias across studies} The following includes a summary of findings, an effect size interpretation, and a grading of the certainty of evidence (table 2). ${ }^{5961}$

\section{Physical activity}

The standardised mean difference for physical activity of 0.42 ( 0.28 to 0.55 ) equates to a moderate effect that translates to a weighted mean difference of 1235 (823 to 1617) daily steps, with more steps in the intervention groups. Certainty in the effect estimate was rated as low. The considerable amount of heterogeneity $\left(\mathrm{I}^{2}=81 \%\right)$ could not be explained by any covariates or risk of bias items. Furthermore, the funnel plot asymmetry and findings from the trim and fill method and the Copas selection model suggest an overestimation of the effect, possibly due to publication and small study bias. Therefore, the certainty in the estimate was downgraded owing to inconsistency and the risk of small study bias due to funnel plot asymmetry.

\section{Moderate to vigorous physical activity}

The standardised mean difference for moderate to vigorous physical activity of 0.23 (0.16 to 0.30 ) equates to a small effect that translates to a weighted mean difference of 48.5 (33.8 to 63.3) minutes weekly moderate to vigorous physical activity time more in the intervention groups. Certainty in the effect estimate was rated as moderate. The substantial amount of heterogeneity $\left(\mathrm{I}^{2}=67 \%\right)$ was partially explained by outcome instrument type and risk of bias items. As the risk of bias items were counterintuitively explaining

\begin{tabular}{|c|c|c|c|c|c|c|}
\hline Outcome & $\begin{array}{l}\text { No of } \\
\text { studies }\end{array}$ & $\begin{array}{c}\text { No of } \\
\text { participants }\end{array}$ & \multicolumn{2}{|c|}{$\begin{array}{l}\text { SMD } \\
(95 \% \mathrm{Cl})\end{array}$} & $\begin{array}{c}\text { SMD } \\
(95 \% \mathrm{Cl})\end{array}$ & $\begin{array}{l}1^{2} \\
(\%)\end{array}$ \\
\hline Physical activity & 103 & 12840 & & $\longrightarrow-$ & $0.42(0.28$ to 0.55$)$ & 81 \\
\hline Moderate to vigorous physical activit & ity 63 & 8250 & & $\leftrightarrow-$ & 0.23 (0.16 to 0.30$)$ & 67 \\
\hline Sedentary time & 38 & 5634 & $\rightarrow-$ & & $-0.12(-0.25$ to 0.01$)$ & 66 \\
\hline
\end{tabular}

Fig 3 | Random effects meta-analysis adjusted to Hedges' $\mathrm{g}$ on effect of interventions on physical activity, moderate to vigorous physical activity, and sedentary time. $S M D=$ standardised mean difference 


\begin{tabular}{|c|c|c|c|c|c|}
\hline Outcomes & Anticipated absolute effects* & $\operatorname{SMD}(95 \% \mathrm{Cl})$ & $\begin{array}{l}\text { No of study compari- } \\
\text { sons and participants }\end{array}$ & $\begin{array}{l}\text { Certainty of } \\
\text { evidencet }\end{array}$ & Comments \\
\hline Physical activity & $\begin{array}{l}\text { SMD translates to weighted mean difference of } 1235 \text { ( } 95 \% \mathrm{Cl} 823 \\
\text { to 1617) daily steps, with more steps in intervention groups }\end{array}$ & $\begin{array}{l}0.42(0.28 \text { to } 0.55) \text { in } \\
\text { favour of intervention }\end{array}$ & $\begin{array}{l}103 \text { studies; } 12840 \\
\text { participants }\end{array}$ & Low $\ddagger \S$ & None \\
\hline $\begin{array}{l}\text { Moderate to vigorous } \\
\text { physical activity }\end{array}$ & $\begin{array}{l}\text { SMD translates to weighted mean difference of } 48.5 \text { ( } 33.8 \text { to } 63.3 \text { ) } \\
\text { minutes weekly MVPA, with more time in intervention groups }\end{array}$ & $\begin{array}{l}0.23(0.16 \text { to } 0.30) \text { in } \\
\text { favour of intervention }\end{array}$ & $\begin{array}{l}63 \text { studies; } 8250 \\
\text { participants }\end{array}$ & Moderate§ & None \\
\hline Sedentary time & $\begin{array}{l}\text { SMD translates to weighted mean difference of } 9.9 \text { ( }-0.8 \text { to } 21.8) \\
\text { daily sedentary minutes, with less time in intervention groups }\end{array}$ & $-0.12(-0.25$ to 0.01$)$ & $\begin{array}{l}38 \text { studies; } 5634 \\
\text { participants }\end{array}$ & Moderate & None \\
\hline Adverse events & $\begin{array}{l}224(6.4 \%) \text { adverse events among } 3501 \text { intervention group } \\
\text { participants; } 186(5.5 \%) \text { adverse events among } 3355 \text { control } \\
\text { group participants }\end{array}$ & $\begin{array}{l}\text { Relative risk } 1.1 \\
(0.93 \text { to } 1.30)\end{array}$ & $\begin{array}{l}34 \text { studies; } 6856 \\
\text { participants }\end{array}$ & High & No heterogeneity \\
\hline $\begin{array}{l}\text { Discontinued } \\
\text { intervention (dropout) }\end{array}$ & $\begin{array}{l}931(10.2 \%) \text { of } 9201 \text { intervention group participants discontinued } \\
\text { interventions; } 713(8.5 \%) \text { of } 9201 \text { control group participants } \\
\text { discontinued interventions }\end{array}$ & $\begin{array}{l}\text { Relative risk } 1.1 \\
(0.96 \text { to } 1.20)\end{array}$ & $\begin{array}{l}117 \text { studies; } 17575 \\
\text { participants }\end{array}$ & High & No heterogeneity \\
\hline
\end{tabular}

$\mathrm{Cl}=$ confidence interval; $\mathrm{MVPA}=$ moderate to vigorous physical activity; SMD=standardised mean difference.

*Absolute effects are calculated from SMDs and relevant standard deviation, as described in methods section.

tGrading of Recommendations Assessment and Evaluation (GRADE) Working Group grades of evidence: high certainty (very confident that true effect lies close to estimate of effect); moderate certainty (moderately confident in effect estimate: true effect is likely to be close to estimate of effect, but possibility that it is substantially different exists); low certainty (confidence in effect estimate is limited: true effect may be substantially different from estimate of effect); very low certainty (very little confidence in effect estimate: true effect is likely to be substantially different from estimate of effect).

FDowngraded by one level owing to inconsistency (unexplained heterogeneity).

$\S$ Downgraded by one level owing to publication bias.

IDowngraded by one level owing to imprecision of results.

heterogeneity (high risk of bias studies reported lower effects), no downgrading due to risk of bias was needed. Furthermore, the funnel plot asymmetry and findings from the trim and fill method and the Copas selection model suggest an overestimation of the effect, possibly due to publication and small study bias. In summary, the certainty was downgraded only owing to risk of small study bias due to funnel plot asymmetry.

\section{Sedentary time}

The standardised mean difference for sedentary time of $-0.12(-0.25$ to 0.01$)$ equates to a small effect that translates to a weighted mean difference of 9.9 ( -0.8 to 21.8) daily sedentary minutes less in the intervention groups. Certainty in the effect estimate was rated as moderate. The overall effect estimate was affected by imprecision, as the upper and lower confidence interval limits represent a large effect and no effect, respectively. The substantial amount of heterogeneity $\left(\mathrm{I}^{2}=66 \%\right)$ was partially explained by differences in participant population and country gross national income to a small extent. Thus, the certainty of evidence was downgraded owing to imprecision.

\section{Adverse events and discontinued interventions}

For both the risk of adverse events and risk of discontinuing the intervention, no heterogeneity was present. Thus, no downgrading was needed, and the certainty in the evidence was rated high for both outcomes.

\section{Discussion}

The finding that PAM based interventions effectively enhance physical activity levels was expected from previous systematic reviews on adult outpatients ${ }^{5}$; older adults ${ }^{25}$; overweight participants ${ }^{26}$; patients with chronic obstructive pulmonary disease, ${ }^{27} 28$ multiple sclerosis, ${ }^{29}$ rheumatic or musculoskeletal diseases, ${ }^{30} 31$ cardiometabolic conditions, ${ }^{32173}$ or type 2 diabetes ${ }^{33-35}$; former healthcare patients ${ }^{36}$; patients in cardiac rehabilitation and with cardiovascular disease $\mathrm{e}^{3738}$; and sedentary adults. ${ }^{39} 40$ Previously, related systematic reviews have focused on more narrow modalities, such as step counting alone, ${ }^{41}$ electronic devices or other accelerometers but excluding pedometers, ${ }^{42} 43$ fitness trackers, ${ }^{44}$ or PAMs available to consumers. ${ }^{45}$ Finally, one systematic review has been published with overlapping but not similar aims, which included fewer randomised controlled trials. ${ }^{46}$ Therefore, this is the first systematic review to summarise the entire body of evidence across different patient populations and different types of PAM. Our finding of a moderate effect on physical activity, equivalent to 1235 (95\% confidence interval 823 to 1617) daily steps, and the small effect on moderate to vigorous physical activity, equivalent to 48.5 (33.8 to 63.3) minutes weekly MVPA time, are both clinically relevant. An increase of 1000 daily steps has previously been reported to reduce all cause mortality by $6-36 \% .{ }^{174}$ This association has a non-linear pattern, with the strongest association in people with fewer than 8000 daily steps. ${ }^{175} 176$ Recent results for moderate to vigorous physical activity show the maximal reduction in risk of mortality to be at around 20-25 minutes of daily moderate to vigorous physical activity. ${ }^{177}$ These findings support the conclusions of a systematic review investigating associations between mortality and physical activity levels and including individual data for 36383 participants, in which any physical activity level was associated with a substantially lower risk of mortality compared with the least active group. ${ }^{177}$ Because of the above, the re-expression of the standardised mean difference for moderate to vigorous physical activity as a small effect might be misleading when the translated effect estimate is close to a third of the recommended level of weekly moderate to vigorous physical activity ${ }^{2}$; hence, a small effect on the physical activity behaviour could still very well be highly relevant clinically.

The small effect on sedentary time $(-0.12,-0.25$ to 0.01), which translates to a weighted mean difference 
of 9.9 (-0.8 to 21.8 ) daily sedentary minutes less in the intervention groups, might be found owing to the imprecision of the results. However, even if the estimated effect is close to the true effect size, the result lacks the clinical relevance of physical activity and moderate to vigorous physical activity, as larger effects are probably needed to reduce clinically relevant outcomes significantly. ${ }^{177} 178$ However, as the strongest inverse correlation between physical activity and sedentary time has been found between light physical activity and sedentary time, ${ }^{179}$ the lack of effect might be explained by participants increasing their moderate to vigorous physical activity, and thus also their physical activity, when receiving feedback from PAMs. This explanation remains theoretical, as it is outside the aim of this systematic review and meta-analysis. The lack of a clinically relevant effect on sedentary time is still noteworthy, however, as this systematic review cannot support the use of PAMs to reduce sedentary time alone.

\section{Factors affecting expected effect of physical activity monitoring}

As seen in the subgroup analyses on physical activity, the standardised mean differences for different methodological factors and across participant populations are somewhat comparable. Thus, no clinical or methodological heterogeneity explained the heterogeneity of the results on physical activity sufficiently, and the moderate effect should be expected in most settings, no matter the population of interest.

For moderate to vigorous physical activity, some heterogeneity was explained by studies using selfreported outcome instruments reporting a larger effect than studies using objective outcome instruments. This could be explained by the questionable validity of commonly used physical activity questionnaires for assessing moderate to vigorous physical activity ${ }^{180}$; however, as the randomisation should handle any validity imbalances between groups, and thus the endpoint effect size, this explanation remains theoretical and outside the primary aim of this study. Future studies investigating moderate to vigorous physical activity should include objective outcome instruments, as self-reported outcome instruments might overestimate the effects of the intervention. Other explanations for heterogeneity of the results on moderate to vigorous physical activity include risk of bias items. However, as these explanations remain counterintuitive, with low risk of bias studies having larger effects, they are not discussed any further.

For sedentary time, the heterogeneity of the results was explained to a very small degree by studies with higher gross national income having a greater effect; however, as this result is clinically irrelevant (correlation coefficient 0.01 (0.00 to 0.02) per US $\$ 10000$ increase), the finding is not discussed any further. Lastly, the heterogeneity of the results on sedentary time was also explained by differences in participant populations, with studies including overweight participants reporting a result favouring the control interventions
(0.11, -0.02 to 0.23$)$. This could be due to overweight participants experiencing an ambiguous and even counterproductive influence from feedback from PAMs similar to the findings identified in a small study with young adults with depression or anxiety. ${ }^{181}$ However, as no explanation was found from the baseline body mass index reported in the included studies, the above finding could also be due to chance alone. For the present, the use of PAMs to reduce sedentary time among overweight participants cannot be supported, and the results should be investigated further in future studies. Finally, however, all of these findings are secondary to the aim of this systematic review and meta-analyses and could, therefore, be due to chance alone.

\section{Publication bias}

The effect sizes on physical activity and moderate to vigorous physical activity might both be affected by publication bias and small study bias. The protocol defined trim and fill adjustment added 36 and 22 fictive studies for physical activity and moderate to vigorous physical activity, respectively, and the effect sizes were adjusted to small and clinically irrelevant for physical activity and moderate to vigorous physical activity, respectively. However, an empirical evaluation of the trim and fill adjustment concludes that it might lead to an excessively conservative estimate. ${ }^{58}$ Because of this, we used the non-protocol defined Copas selection model as an alternative sensitivity analysis and adjustment method to the trim and fill method. ${ }^{182} 183$ The Copas selection model supported the trim and fill method for both outcomes, but it was not as conservative on moderate to vigorous physical activity as the former alternative. Statistical evaluation and especially correction of small study bias are complex; even though the expected true effect sizes for physical activity and moderate to vigorous physical activity might be overestimated, the results still suggest and support the use of PAM interventions.

\section{Strengths and limitations of study}

With its 121 included studies, this study stands as the largest available systematic review and meta-analysis investigating the effect of PAMs on physical activity behaviour. Furthermore, it includes all populations (healthy individuals and patients) and provides precise estimates of the effect sizes for physical activity and moderate to vigorous physical activity. The following limitations should, however, be considered when interpreting the results. Firstly, a substantial amount of heterogeneity was found for all outcomes and explained to some degree only for moderate to vigorous physical activity and sedentary time. Secondly, studies conducted in high income countries are overrepresented, as only eight studies were conducted in non-high income countries, all upper middle income countries. ${ }^{128087104123129150156}$ Consequently, the external validity of our results is limited to high income countries. Thirdly, studies with a high female participation rate are overrepresented, limiting the external validity to female populations. However, as 
the percentage of female participants in the studies did not explain any heterogeneity, the influence of gender seems to be low. Fourthly, the studies all used objective PAMs as intervention tools but included a variety of physical activity outcome instruments, including selfreported measures (according to appendix 3, 42\% of comparisons for physical activity, $45 \%$ for moderate to vigorous physical activity, and $48 \%$ for sedentary time), which also explained some heterogeneity for moderate to vigorous physical activity. As previously discussed, self-reported outcome measures have a low validity for moderate to vigorous physical activity, which might explain the finding. The inclusion of studies using self-reported measures might limit our results; however, as self-reported measures seem to affect the effect estimate only for moderate to vigorous physical activity, the inclusion could also be seen as a strength for physical activity and sedentary time. Finally, some patient populations appear in only a few studies and thus few participants (cardiovascular, musculoskeletal, neurological, psychiatric, and pulmonary patient populations). This limits the generalisability to these populations, as the effect sizes might be driven by other populations. But as no evidence indicates that these populations will react differently to feedback from PAMs or suggests that they will have negative or unexpected effects, the results should apply equally.

\section{Implications for practice and research}

The certainty of the evidence for a moderate effect on physical activity was rated low and could very well be changed by future studies. The effect estimate might also be overestimated owing to publication bias and small study bias. The certainty of the evidence for a small effect on moderate to vigorous physical activity was rated moderate, and it is likely to be close to the true effect. However, the effect estimate might also be overestimated owing to publication bias and small study bias and affected by studies with selfreported instruments reporting larger effects. However, according to the cumulative meta-analysis, the effect estimates on physical activity have been stable for some years, which should inform future studies and encourage comparisons only where needed (for example, large scale studies on physical activity and moderate to vigorous physical activity to avoid small study bias and studies on sedentary time in general and especially among overweight adults).

Even though the effects might be overestimated, clinically relevant effects on physical activity and moderate to vigorous physical activity should be expected when implementing PAMs among healthy or patient populations. These perspectives and recommendations, however, do not apply to studies investigating the effects on sedentary time, as we provide an imprecise effect estimate with low clinical relevance on sedentary time. Because of this, future investigations on sedentary time are encouraged.

In summary, this systematic review and metaanalysis summarises the results of 121 randomised controlled trials and is the first large summary to conclude that although individual studies might show different results, this is most likely due to the expected heterogeneity of the effects among individuals and not because one type of PAM intervention is superior to others. In the future, researchers should investigate how PAMs can be used in combination with other behavioural change contents or how PAMs might affect sedentary time.

\section{Conclusions}

This systematic review and meta-analysis identified 121 studies with 141 comparisons investigating the effect of PAMs. We summarise the results from 16743 unique participants and provide low certainty of evidence for a moderate effect on physical activity equal to 1235 daily steps more, moderate certainty of evidence for a small effect on moderate to vigorous physical activity equal to 48.5 minutes weekly moderate to vigorous physical activity time more, and moderate certainty of evidence for a small but insignificant effect on sedentary time equal to 9.9 daily sedentary minutes less in the intervention groups. Because of the certainty of evidence, future studies could change the overall estimates and are encouraged to investigate how PAMs can be used in combination with other interventions or how PAMs can be used to reduce sedentary time.

\section{AUTHOR AFFILIATIONS}

${ }^{1}$ Department of Public Health, Section of Social Medicine, University of Copenhagen, Copenhagen, Denmark

${ }^{2}$ Department of Occupational Therapy and Physiotherapy, Copenhagen University Hospital, Rigshospitalet Copenhagen, Denmark

${ }^{3}$ Department of Brain Injury Rehabilitation, Copenhagen University Hospital, Rigshospitalet, Denmark

${ }^{4}$ Parker Institute, Bispebjerg and Frederiksberg Hospital, Capital Region, Frederiksberg, Denmark

${ }^{5}$ Danish Health Authority, Copenhagen, Denmark

${ }^{6}$ Danish Cancer Society Research Center, Danish Cancer Society, Copenhagen, Denmark

${ }^{7}$ Research Unit of Musculoskeletal Function and Physiotherapy, Department of Sports Science and Clinical Biomechanics, University of Southern Denmark, Odense, Denmark

${ }^{8}$ Department of Physiotherapy and Occupational Therapy, Copenhagen University Hospital, Herlev and Gentofte, Denmark ${ }^{9}$ Section of Health Services Research, Department of Public Health, University of Copenhagen, Denmark

We thank Andreas Lund Hessner for the help with data collection. Contributors: RTL and JC conceived the project. CBJ and HL supervised the work. RTL, JC, and CBJ discussed and planned the analysis. RTL collected the data from online sources, and JC checked this work. VW, CBK, CK, RTL, and JC independently reviewed trials for meeting of inclusion criteria and extracted data. VW contacted corresponding authors by email to request additional data. RTL did the analysis. RTL, JC, and CBJ contributed to the interpretation of results and writing of the discussion section. All authors discussed the results and contributed to the writing and editing of the manuscript. RTL and JC are the guarantors. The corresponding author attests that all listed authors meet authorship criteria and that no others meeting the criteria have been omitted.

Funding: The content presented in this paper was produced as part of the project REACH, which has received funding from the European Union's Horizon 2020 research and innovation programme under grant agreement number 690425 (reach2020.eu). The funders had no role in considering the study design, in the collection, analysis, or interpretation of data, in the writing of the report, or in the decision to submit the article for publication. 
Competing interests: All authors have completed the ICMJE uniform disclosure form at www.icmje.org/disclosure-of-interest/ and declare: support from the European Union's Horizon 2020 research and innovation programme for the submitted work; no financial relationships with any organisations that might have an interest in the submitted work in the previous three years; no other relationships or activities that could appear to have influenced the submitted work.

Ethical approval: No individual level data are included in this manuscript. All data are aggregated data from clinical trials.

Data sharing: The statistical analysis plan and dataset can be available from the corresponding author on reasonable request. The lead authors (the manuscript's guarantors) affirm that the manuscript is an honest, accurate, and transparent account of the study being reported; that no important aspects of the study have been omitted; and that any discrepancies from the study as planned (and, if relevant, registered) have been explained.

Dissemination to participants and related patient and public communities: Results will be disseminated using social media such as Linkedln, at international conferences such as the Scandinavian Sports Medicine Congress, and to relevant stakeholders at non-profit organisations and within healthcare.

Provenance and peer review: Not commissioned; externally peer reviewed.

This is an Open Access article distributed in accordance with the Creative Commons Attribution Non Commercial (CC BY-NC 4.0) license, which permits others to distribute, remix, adapt, build upon this work non-commercially, and license their derivative works on different terms, provided the original work is properly cited and the use is noncommercial. See: http://creativecommons.org/licenses/by-nc/4.0/.

1 Lee IM, Shiroma EJ, Lobelo F, Puska P, Blair SN, Katzmarzyk PT, Lancet Physical Activity Series Working Group. Effect of physical inactivity on major non-communicable diseases worldwide: an analysis of burden of disease and life expectancy. Lancet 2012;380:219-29. doi:10.1016/S0140-6736(12)61031-9

2 World Health Organization. WHO guidelines on physical activity and sedentary behaviour. 2020. https://apps.who.int/iris/ handle/10665/336656

3 Stunkard A. A Method of Studying Physical Activity in Man. Am I Clin Nutr 1960;8:595-601. doi:10.1093/ajcn/8.5.595

4 Patel MS, Asch DA, Volpp KG. Wearable devices as facilitators, not drivers, of health behavior change. JAMA 2015:313:459-60. doi:10.1001/jama.2014.14781

5 Bravata DM, Smith-Spangler C, Sundaram V, et al. Using pedometers to increase physical activity and improve health: a systematic review. JAMA 2007;298:2296-304. doi:10.1001/jama.298.19.2296

6 Al-Anqodi N, McCullough F, Salter AM. Feasibility of smartphone application to promote physical activity in healthy Omani female adults. Proc Nutr Soc 2018;77. doi:10.1017/ S0029665118001556

7 Ashton LM, Morgan PJ, Hutchesson MJ, Rollo ME, Collins CE. Feasibility and preliminary efficacy of the 'HEYMAN' healthy lifestyle program for young men: a pilot randomised controlled trial. Nutr J 2017;16:2. doi:10.1186/s12937-017-0227-8

8 Backman M, Wengström Y, Johansson B, et al. A randomized pilot study with daily walking during adjuvant chemotherapy for patients with breast and colorectal cancer. Acta Oncol 2014;53:510-20. doi:1 $0.3109 / 0284186 \times .2013 .873820$

9 Eisenberg MH, Phillips LA, Fowler L, Moore PJ. The Impact of E-diaries and Accelerometers on Young Adults' Perceived and Objectively Assessed Physical Activity. Psychol Sport Exerc 2017;30:55-63. doi:10.1016/.jpsychsport.2017.01.008

10 Kim KA, Hwang SY. [Effects of a Daily Life-Based Physical Activity Enhancement Program for Middle-Aged Women at Risk for Cardiovascular Disease]. / Korean Acad Nurs 2019;49:113-25. doi:10.4040/jkan.2019.49.2.113

11 Motl RW, Dlugonski D, Pilutti LA, Klaren RE. Does the effect of a physical activity behavioral intervention vary by characteristics of people with multiple sclerosis?!nt I MS Care 2015;17:65-72. doi:10.7224/1537-2073.2014-016

12 Ribeiro MA, Martins MA, Carvalho CRF. Interventions to increase physical activity in middle-age women at the workplace: a randomized controlled trial. Med Sci Sports Exerc 2014;46:1008-15. doi:10.1249/MSS.0000000000000190

13 Ryan JM, Fortune J, Stennett A, et al. Changing physical activity behaviour for people with multiple sclerosis: protocol of a randomised controlled feasibility trial (iStep-MS). BMJ Open 2017;7: e018875

14 Thompson WG, Koepp GA, Levine JA. Increasing physician activity with treadmill desks. Work 2014;48:47-51. doi:10.3233/WOR131708
15 Whelan ME, Orme MW, Kingsnorth AP, Sherar LB, Denton FL, Esliger DW. Examining the Use of Glucose and Physical Activity SelfMonitoring Technologies in Individuals at Moderate to High Risk of Developing Type 2 Diabetes: Randomized Trial. JMIR Mhealth Uhealth 2019;7:e14195. doi:10.2196/14195

16 Peacock AS, Bogossian FE, Wilkinson SA, Gibbons KS, Kim C, McIntyre HD. A Randomised Controlled Trial to Delay or Prevent Type 2 Diabetes after Gestational Diabetes: Walking for Exercise and Nutrition to Prevent Diabetes for You. Int / Endocrinol 2015;2015:423717. doi:10.1155/2015/423717

17 Martin SS, Feldman DI, Blumenthal RS, et al. mActive: A Randomized Clinical Trial of an Automated mHealth Intervention for Physical Activity Promotion. / Am Heart Assoc 2015;4:e002239. doi:10.1161/JAHA.115.002239

18 Holmes VA, Draffin CR, Patterson CC, et al, PAIGE Study Group. Postnatal Lifestyle Intervention for Overweight Women With Previous Gestational Diabetes: A Randomized Controlled Trial. J Clin Endocrinol Metab 2018;103:2478-87. doi:10.1210/jc.2017-02654

19 Eastep E, Beveridge S, Eisenman P, Ransdell L, Shultz B. Does augmented feedback from pedometers increase adults' walking behavior?Percept Mot Skills 2004;99:392-402. doi:10.2466/ pms.99.2.392-402

20 Maselli M, Gobbi E, Carraro A. Effectiveness of individual counseling and activity monitors to promote physical activity among university students. J Sports Med Phys Fitness 2019;59:132-40.

21 Reijonsaari K, Vehtari A, Kahilakoski OP, van Mechelen W, Aro T, Taimela S. The effectiveness of physical activity monitoring and distance counseling in an occupational setting - results from a randomized controlled trial (CoAct). BMC Public Health 2012;12:344. doi:10.1186/1471-2458-12-344

22 Pope ZC, Barr-Anderson DJ, Lewis BA, Pereira MA, Gao Z. Use of wearable technology and social media to improve physical activity and dietary behaviors among college students: A 12-week randomized pilot study. Int J Environ Res Public Health 2019;16:3579. doi:10.3390/ijerph16193579

23 Slootmaker SM, Chinapaw MJ, Schuit Al, Seidell JC, Van Mechelen W. Feasibility and effectiveness of online physical activity advice based on a personal activity monitor: randomized controlled trial. J Med Internet Res 2009;11:e27. doi:10.2196/jmir.1139

24 Van Blarigan EL, Chan H, Van Loon K, et al. Self-monitoring and reminder text messages to increase physical activity in colorectal cancer survivors (Smart Pace): a pilot randomized controlled trial. BMC Cancer 2019:19:218. doi:10.1186/s12885-019-5427-5

25 Larsen RT, Christensen J, Juhl CB, Andersen HB, Langberg H. Physical activity monitors to enhance amount of physical activity in older adults - a systematic review and meta-analysis. Eur Rev Aging Phys Act 2019;16:7. doi:10.1186/s11556-019-0213-6

26 de Vries HJ, Kooiman TJM, van Ittersum MW, van Brussel M, de Groot M. Do activity monitors increase physical activity in adults with overweight or obesity? A systematic review and meta-analysis. Obesity (Silver Spring) 2016;24:2078-91. doi:10.1002/oby.21619

27 Qiu S, Cai X, Wang X, et al. Using step counters to promote physical activity and exercise capacity in patients with chronic obstructive pulmonary disease: a meta-analysis. Ther Adv Respir Dis 2018;12:1753466618787386. doi:10.1177/1753466618787386

28 Armstrong M, Winnard A, Chynkiamis N, Boyle S, Burtin C, Vogiatzis I. Use of pedometers as a tool to promote daily physical activity levels in patients with COPD: a systematic review and meta-analysis. Eur Respir Rev 2019:28:190039. doi:10.1183/16000617.0039-2019

29 Rintala A, Hakala S, Paltamaa J, Heinonen A, Karvanen J, Sjögren T. Effectiveness of technology-based distance physical rehabilitation interventions on physical activity and walking in multiple sclerosis: a systematic review and meta-analysis of randomized controlled trials. Disabil Rehabil 2018;40:373-87. doi:10.1080/09638288.2016.12 60649

30 Mansi S, Milosavljevic S, Baxter GD, Tumilty S, Hendrick P. A systematic review of studies using pedometers as an intervention for musculoskeletal diseases. BMC Musculoskelet Disord 2014:15:231. doi:10.1186/1471-2474-15-231

31 Davergne T, Pallot A, Dechartres A, Fautrel B, Gossec L. Use of Wearable Activity Trackers to Improve Physical Activity Behavior in Patients With Rheumatic and Musculoskeletal Diseases: A Systematic Review and Meta-Analysis. Arthritis Care Res (Hoboken) 2019;71:758-67. doi:10.1002/acr.23752

32 Kirk MA, Amiri M, Pirbaglou M, Ritvo P. Wearable Technology and Physical Activity Behavior Change in Adults With Chronic Cardiometabolic Disease: A Systematic Review and Meta-Analysis. Am J Health Promot 2019;33:778-91. doi:10.1177/0890117118816278

33 Vaes AW, Cheung A, Atakhorrami M, et al. Effect of 'activity monitorbased' counseling on physical activity and health-related outcomes in patients with chronic diseases: A systematic review and metaanalysis. Ann Med 2013;45:397-412. doi:10.3109/07853890.201 3.810891 
34 Funk M, Taylor EL. Pedometer-based walking interventions for freeliving adults with type 2 diabetes: a systematic review. Curr Diabetes Rev 2013;9:462-71. doi:10.2174/15733998113096660084

35 Baskerville R, Ricci-Cabello I, Roberts N, Farmer A. Impact of accelerometer and pedometer use on physical activity and glycaemic control in people with Type 2 diabetes: a systematic review and meta-analysis. Diabet Med 2017;34:612-20. doi:10.1111/ dme.13331

36 Braakhuis HEM, Berger MAM, Bussmann JBJ. Effectiveness of healthcare interventions using objective feedback on physical activity: A systematic review and meta-analysis. J Rehabil Med 2019:51:151-9. doi:10.2340/16501977-2522

37 Hannan AL, Harders MP, Hing W, Climstein M, Coombes JS, Furness J. Impact of wearable physical activity monitoring devices with exercise prescription or advice in the maintenance phase of cardiac rehabilitation: systematic review and meta-analysis. BMC Sports Sci Med Rehabil 2019;11:14. doi:10.1186/s13102-019-0126-8

38 Kanejima Y, Kitamura M, Izawa KP. Self-monitoring to increase physical activity in patients with cardiovascular disease: a systematic review and meta-analysis. Aging Clin Exp Res 2019;31:163-73. doi:10.1007/s40520-018-0960-7

39 Qiu S, Cai X, Ju C, et al. Step Counter Use and Sedentary Time in Adults: A Meta-Analysis. Medicine (Baltimore) 2015:94:e1412. doi:10.1097/MD.0000000000001412

40 Stephenson A, McDonough SM, Murphy MH, Nugent CD, Mair JL. Using computer, mobile and wearable technology enhanced interventions to reduce sedentary behaviour: a systematic review and meta-analysis. Int J Behav Nutr Phys Act 2017;14:105. doi:10.1186/ s12966-017-0561-4

41 Chaudhry UAR, Wahlich C, Fortescue R, Cook DG, Knightly R, Harris T. The effects of step-count monitoring interventions on physical activity: systematic review and meta-analysis of communitybased randomised controlled trials in adults. Int J Behav Nutr Phys Act 2020;17:129. doi:10.1186/s12966-020-01020-8

42 Lewis ZH, Lyons EJ, Jarvis JM, Baillargeon J. Using an electronic activity monitor system as an intervention modality: A systematic review. BMC Public Health 2015;15:585. doi:10.1186/s12889-015-1947-3

43 Goode AD, Reeves MM, Eakin EG. Telephone-delivered interventions for physical activity and dietary behavior change: an updated systematic review. Am J Prev Med 2012;42:81-8. doi:10.1016/j. amepre.2011.08.025

44 Lynch C, Bird S, Lythgo N, Selva-Raj I. Changing the Physical Activity Behavior of Adults With Fitness Trackers: A Systematic Review and Meta-Analysis. Am J Health Promot 2020;34:418-30. doi:10.1177/0890117119895204

45 Brickwood K-J, Watson G, O'Brien J, Williams AD ConsumerBased Wearable Activity Trackers Increase Physical Activity Participation: Systematic Review and Meta-Analysis. JMIR Mhealth Whealth 2019:7:e11819. doi:10.2196/11819

46 Hakala S, Rintala A, Immonen J, Karvanen J, Heinonen A, Sjögren T. Effectiveness of physical activity promoting technology-based distance interventions compared to usual care. Systematic review, meta-analysis and meta-regression. Eur I Phys Rehabil Med 2017;53:953-67. doi:10.23736/S1973-9087.17.04585-3

47 Higgins JPT, Thomas J, Chandler J, et al, eds. Cochrane Handbook for Systematic Reviews of Interventions. 2021. https://training.cochrane. org/handbook/current.

48 Shamseer L, Moher D, Clarke M, et al, PRISMA-P Group. Preferred reporting items for systematic review and meta-analysis protocols (PRISMA-P) 2015: elaboration and explanation. BMJ 2015;350:g7647. doi:10.1136/bmj.g7647

49 Larsen RT, Wagner V, Keller C, Juhl CB, Langberg H, Christensen J. Feedback from physical activity monitors to enhance amount of physical activity in adults-a protocol for a systematic review and meta-analysis. Syst Rev 2019;8:53. doi:10.1186/s13643-019 0970-3

50 Hall M, Juhl CB, Lund H, Thorlund JB. Knee Extensor Muscle Strength in Middle-Aged and Older Individuals Undergoing Arthroscopic Partial Meniscectomy: A Systematic Review and Meta-Analysis. Arthritis Care Res (Hoboken) 2015;67:1289-96. doi:10.1002/ acr.22581

51 Sterne JAC, Savović J, Page MJ, et al. RoB 2: a revised tool for assessing risk of bias in randomised trials. BM/ 2019;366:14898. doi:10.1136/bmj.l4898

52 Higgins JPT, Thomas J, Chandler J, et al, eds. Cochrane Handbook for Systematic Reviews of Interventions: Chapter 5: Collecting data. 2021. https://training.cochrane.org/handbook/current/chapter-05.

53 IntHout J, loannidis JP, Borm GF. The Hartung-Knapp-Sidik-Jonkman method for random effects meta-analysis is straightforward and considerably outperforms the standard DerSimonian-Laird method. BMC Med Res Methodol 2014;14:25. doi:10.1186/1471-228814-25

54 Lin L, Aloe AM. Evaluation of various estimators for standardized mean difference in meta-analysis. Stat Med 2021;40:403-26. doi:10.1002/sim.8781
55 Friedrich JO, Adhikari NKJ, Beyene J. Inclusion of zero total event trials in meta-analyses maintains analytic consistency and incorporates all available data. BMC Med Res Methodol 2007;7:5. doi:10.1186/1471-2288-7-5

56 Duval S, Tweedie R. Trim and fill: A simple funnel-plot-based method of testing and adjusting for publication bias in metaanalysis. Biometrics 2000;56:455-63. doi:10.1111/j.0006341X.2000.00455.x

57 Higgins JPT, Thomas J, Chandler J, et al, eds. Cochrane Handbook for Systematic Reviews of Interventions: Chapter 10: Addressing reporting biases. 2011. https://handbook-5-1.cochrane.org/ chapter_10/10_4_4_2 trim and fill.htm.

58 Schwarzer G, Carpenter J, Rücker G. Empirical evaluation suggests Copas selection model preferable to trim-and-fill method for selection bias in meta-analysis. J Clin Epidemiol 2010;63:282-8. doi:10.1016/j.jclinepi.2009.05.008

59 Guyatt GH, Oxman AD, Vist GE, et al, GRADE Working Group. GRADE: an emerging consensus on rating quality of evidence and strength of recommendations. BMJ 2008;336:924-6. doi:10.1136/ bmj.39489.470347.AD

60 Schünemann H, Brożek J, Guyatt G, Oxman A. GRADE Handbook. 2013. http://gdt.guidelinedevelopment.org/central_prod/_design/ client/handbook/handbook.html\#h.1i2bwkm8zpjo.

61 Higgins JPT, Thomas J, Chandler J, et al, eds. Cochrane Handbook for Systematic Reviews of Interventions: Chapter 12: Interpreting results and drawing conclusions. 2011. https://handbook-5-1.cochrane.org/ chapter_12/12_6_2_re_expressing_smds_using_rules_of_thumb_ for_effect_sizes.htm.

62 Higgins JPT, Thomas J, Chandler J, et al, eds. Cochrane Handbook for Systematic Reviews of Interventions: Chapter 22: Prospective approaches to accumulating evidence. 2021. https://training. cochrane.org/handbook/current/chapter-22\#section-22-4-2.

63 World Bank. World Bank Country and Lending Groups. 2020. https:// datahelpdesk.worldbank.org/knowledgebase/articles/906519world-bank-country-and-lending-groups.

64 Aittasalo M, Rinne M, Pasanen M, Kukkonen-Harjula K, Vasankari T. Promoting walking among office employees - evaluation of a randomized controlled intervention with pedometers and e-mail messages. BMC Public Health 2012;12:403. doi:10.1186/14712458-12-403

65 Armit CM, Brown WJ, Marshall AL, et al. Randomized trial of three strategies to promote physical activity in general practice. Prev Med 2009:48:156-63 doi:10.1016/i.ypmed 2008.11.009

66 Arrogi A, Bogaerts A, Seghers J, et al. Evaluation of stAPP: a smartphone-based intervention to reduce prolonged sitting among Belgian adults. Health Promot Int 2019;34:16-27. doi:10.1093/ heapro/dax046

67 Baker G, Gray SR, Wright A, et al, Scottish Physical Activity Research Collaboration (SPARColl). The effect of a pedometer-based community walking intervention "Walking for Wellbeing in the West" on physical activity levels and health outcomes: a 12-week randomized controlled trial. Int J Behav Nutr Phys Act 2010;7:51. doi:10.1186/1479-5868-7-51

68 Baker G, Mutrie N, Lowry R. A comparison of goals set in steps using a pedometer and goals set in minutes: a randomized controlled trial. Int J Health Promot Educ 2011;49:60-8. doi:10.1080/14635240.20 11.10708210.

69 Baker G, Mutrie N, Lowry R. Using pedometers as motivational tools: Are goals set in steps more effective than goals set in minutes for increasing walking? Int I Health Promot Educ 2008:46:21-6. doi:10.1 080/14635240.2008.10708123

70 Barwais FA, Cuddihy TF, Tomson LM. Physical activity, sedentary behavior and total wellness changes among sedentary adults: a 4-week randomized controlled trial. Health Qual Life Outcomes 2013;11:183. doi:10.1186/1477-7525-11-183

71 Basen-Engquist K, Taylor CLC, Rosenblum C, et al. Randomized pilot test of a lifestyle physical activity intervention for breast cancer survivors. Patient Educ Couns 2006;64:225-34. doi:10.1016/j. pec.2006.02.006

72 Berglind D, Yacaman-Mendez D, Lavebratt C, Forsell Y. The Effect of Smartphone Apps Versus Supervised Exercise on Physical Activity, Cardiorespiratory Fitness, and Body Composition Among Individuals With Mild-to-Moderate Mobility Disability: Randomized Controlled Trial. JMIR Mhealth Uhealth 2020;8:e14615. doi:10.2196/14615

73 Biddle SJH, Edwardson CL, Wilmot EG, et al. A Randomised Controlled Trial to Reduce Sedentary Time in Young Adults at Risk of Type 2 Diabetes Mellitus: Project STAND (Sedentary Time ANd Diabetes). PLoS One 2015;10:e0143398. doi:10.1371/journal.pone.0143398

74 Bond DS, Vithiananthan S, Thomas JG, et al. Bari-Active: a randomized controlled trial of a preoperative intervention to increase physical activity in bariatric surgery patients. Surg Obes Relat Dis 2015;11:169-77. doi:10.1016/j.soard.2014.07.010

75 Butler L, Dwyer D. Pedometers may not provide a positive effect on walking activity. Health Promot J Austr 2004;2004:134-6. doi:10.1071/HE04134 
76 Cadmus-Bertram L, Tevaarwerk AJ, Sesto ME, Gangnon R, Van Remortel B, Date P. Building a physical activity intervention into clinical care for breast and colorectal cancer survivors in Wisconsin: randomized controlled pilot trial. J Cancer Surviv 2019;13:593-602. doi:10.1007/s11764-019-00778-6

77 Carr LJ, Karvinen K, Peavler M, Smith R, Cangelosi K. Multicomponent intervention to reduce daily sedentary time: a randomised controlled trial. BMJ Open 2013;3:e003261. doi:10.1136/ bmjopen-2013-003261

78 Cheung NW, Blumenthal C, Smith BJ, et al. A Pilot Randomised Controlled Trial of a Text Messaging Intervention with Customisation Using Linked Data from Wireless Wearable Activity Monitors to Improve Risk Factors Following Gestational Diabetes. Nutrients 2019;11:E590. doi:10.3390/nu11030590

79 Cheung P, Chen S. Emmy Man Yee Wong. Using Mobile Phone Messages in Pedometer-Based Intervention for Working Adults in Hong Kong. Asian J Exerc Sports Sci 2012;9:76-85.

80 Coelho CM, Reboredo MM, Valle FM, et al. Effects of an unsupervised pedometer-based physical activity program on daily steps of adults with moderate to severe asthma: a randomized controlled trial. J Sports Sci 2018;36:1186-93. doi:10.1080/02640414.2017.1364402

81 Cooper AJ, Dearnley K, Williams KM, et al. Protocol for Get Moving: a randomised controlled trial to assess the effectiveness of three minimal contact interventions to promote fitness and physical activity in working adults. BMC Public Health 2015;15:296. doi:10.1186/ s12889-015-1654-0

82 Creel DB, Schuh LM, Reed CA, et al. A randomized trial comparing two interventions to increase physical activity among patients undergoing bariatric surgery. Obesity (Silver Spring) 2016;24:16608. doi:10.1002/oby.21548

83 Dadaczynski K, Schiemann S, Backhaus O. Promoting physical activity in worksite settings: results of a German pilot study of the online intervention Healingo fit. BMC Public Health 2017:17:696. doi:10.1186/s12889-017-4697-6

84 Dicken-Kano R, Bell MM. Pedometers as a means to increase walking and achieve weight loss. J Am Board Fam Med 2006;19:524-5. doi:10.3122/jabfm.19.5.524

85 DuVall C, Dinger MK, Taylor EL, Bemben D. Minimal-contact physical activity interventions in women: a pilot study. Am J Health Behav 2004:28:280-6. doi:10.5993/AlHB.28.3.9

86 Finkelstein EA, Haaland BA, Bilger M, et al. Effectiveness of activity trackers with and without incentives to increase physical activity (TRIPPA): a randomised controlled trial. Lancet Diabetes Endocrinol 2016;4:983-95. doi:10.1016/S2213-8587(16)30284-4

87 Freitas PD, Silva AG, Ferreira PG, et al. Exercise Improves Physical Activity and Comorbidities in Obese Adults with Asthma. Med Sci Sports Exerc 2018;50:1367-76. doi:10.1249/ MSS.0000000000001574

88 Glynn LG, Hayes PS, Casey M, et al. Effectiveness of a smartphone application to promote physical activity in primary care: the SMART MOVE randomised controlled trial. Br J Gen Pract 2014;64:e384-9. doi:10.3399/bjgp14X680461

89 Gokal K, Wallis D, Ahmed S, Boiangiu I, Kancherla K, Munir F. Effects of a self-managed home-based walking intervention on psychosocial health outcomes for breast cancer patients receiving chemotherapy: a randomised controlled trial. Support Care Cancer 2016;24:113966. doi:10.1007/s00520-015-2884-5

90 Goto M, Takedani H, Haga N, et al. Self-monitoring has potential for home exercise programmes in patients with haemophilia. Haemophilia 2014:20:e121-7. doi:10.1111/hae.12355

91 Greene J, Sacks R, Piniewski B, Kil D, Hahn JS. The impact of an online social network with wireless monitoring devices on physical activity and weight loss. J Prim Care Community Health 2013;4:189-94. doi:10.1177/2150131912469546

92 Grey EB, Thompson D, Gillison FB. Effects of a Web-Based, Evolutionary Mismatch-Framed Intervention Targeting Physical Activity and Diet: a Randomised Controlled Trial. Int J Behav Med 2019;26:645-57. doi:10.1007/s12529-019-09821-3

93 Hardeman W, Mitchell J, Pears S, et al, VBI Research Team. Evaluation of a very brief pedometer-based physical activity intervention delivered in NHS Health Checks in England: The VBI randomised controlled trial. PLoS Med 2020;17:e1003046. doi:10.1371/ journal.pmed.1003046

94 Harrington DM, Champagne CM, Broyles ST, Johnson WD, TudorLocke C, Katzmarzyk PT. Steps ahead: a randomized trial to reduce unhealthy weight gain in the Lower Mississippi Delta. Obesity (Silver Spring) 2014;22:E21-8. doi:10.1002/oby.20684

95 Hartman SJ, Nelson SH, Cadmus-Bertram LA, Patterson RE, Parker BA, Pierce JP. Technology- and Phone-Based Weight Loss Intervention: Pilot RCT in Women at Elevated Breast Cancer Risk. Am I Prev Med 2016;51:714-21. doi:10.1016/j.amepre.2016.06.024

96 Hultquist CN, Albright C, Thompson DL. Comparison of walking recommendations in previously inactive women. Med Sci Sports Exerc 2005;37:676-83. doi:10.1249/01.MSS.0000158993.39760.1B
97 Hurling R, Catt M, Boni MD, et al. Using internet and mobile phone technology to deliver an automated physical activity program: randomized controlled trial. / Med Internet Res 2007:9:e7. doi:10.2196/jmir.9.2.e7

98 Jakicic JM, Davis KK, Rogers RJ, et al. Effect of Wearable Technology Combined With a Lifestyle Intervention on Long-term Weight Loss: The IDEA Randomized Clinical Trial. JAMA 2016;316:1161-71 doi:10.1001/jama.2016.12858

99 Katzmarzyk PT, Champagne CM, Tudor-Locke C, et al. A short-term physical activity randomized trial in the Lower Mississippi Delta. PLoS One 2011;6:e26667. doi:10.1371/journal.pone.0026667

100 Kendzor DE, Allicock M, Businelle MS, Sandon LF, Gabriel KP, Frank SG. Evaluation of a Shelter-Based Diet and Physical Activity Intervention for Homeless Adults. J Phys Act Health 2017;14:88-97. doi:10.1123/jpah.2016-0343

101 Kim C, Draska M, Hess ML, Wilson EJ, Richardson CR. A web-based pedometer programme in women with a recent history of gestational diabetes. Diabet Med 2012;29:278-83. doi:10.1111/j.14645491.2011.03415.

102 Kim JY, Lee MK, Lee DH, et al. Effects of a 12-week home-based exercise program on quality of life, psychological health, and the level of physical activity in colorectal cancer survivors: a randomized controlled trial. Support Care Cancer 2019;27:2933-40. doi:10.1007/s00520-018-4588-0

103 Kitagawa T, Higuchi Y, Todo E, Ueda T, Ando S, Murakami T. Tailored feedback reduced prolonged sitting time and improved the health of housewives: a single-blind randomized controlled pilot study. Women Health 2020;60:212-2 . doi:10.1080/03630242.2019.1616043

104 Kovelis D, Zabatiero J, Furlanetto KC, Mantoani LC, Proença M, Pitta F. Short-term effects of using pedometers to increase daily physical activity in smokers: a randomized trial. Respir Care 2012;57:108997. doi: $10.4187 /$ respcare. 01458

105 Li LC, Sayre EC, Xie H, Clayton C, Feehan LM. A Community-Based Physical Activity Counselling Program for People With Knee Osteoarthritis: Feasibility and Preliminary Efficacy of the Track OA Study. JMIR Mhealth Uhealth 2017;5:e86. doi:10.2196/ mhealth.7863

106 Ligibel JA, Meyerhardt J, Pierce JP, et al. Impact of a telephone-based physical activity intervention upon exercise behaviors and fitness in cancer survivors enrolled in a cooperative group setting. Breast Cancer Res Treat 2012;132:205-13. doi:10.1007/s10549-0111882-7

107 Long JE, Ring C, Bosch JA, et al. A life-style physical activity intervention and the antibody response to pneumococcal vaccination in women. Psychosom Med 2013;75:774-82. doi:10.1097/ PSY.0b013e3182a0b664

108 Mansi S, Milosavljevic S, Tumilty S, Hendrick P, Higgs C, Baxter DG. Investigating the effect of a 3-month workplace-based pedometerdriven walking programme on health-related quality of life in meat processing workers: a feasibility study within a randomized controlled trial. BMC Public Health 2015;15:410. doi:10.1186/s12889-015 $1736-2$

109 Matevey C, Rogers LQ Dawson E, et al. Lack of Reactivity During Pedometer Self-Monitoring in Adults. Meas Phys Educ Exerc Sci 2006;10:1-11. doi:10.1207/s15327841mpee1001_

110 Melton BF Buman MP Vogel RL et al Wearable Devices to Improve Physical Activity and Sleep: A Randomized Controlled Trial of CollegeAged African American Women. J Black Stud 2016;47:610-25. doi:10.1177/0021934716653349

111 Merom D, Rissel C, Phongsavan P, et al. Promoting walking with pedometers in the community: the step-by-step trial. Am J Prev Med 2007;32:290-7. doi:10.1016/j.amepre.2006.12.007

112 Miragall M, Domínguez-Rodríguez A, Navarro J, Cebolla A, Baños RM. Increasing physical activity through an Internet-based motivational intervention supported by pedometers in a sample of sedentary students: A randomised controlled trial. Psychol Health 2018;33:465-82. doi:10.1080/08870446.2017.1368511

113 Morgan PJ, Callister R, Collins CE, et al. The SHED-IT community trial: a randomized controlled trial of internet- and paper-based weight oss programs tailored for overweight and obese men. Ann Behav Med 2013;45:139-52. doi:10.1007/s12160-012-9424-z

114 Murawski B, Plotnikoff RC, Rayward AT, et al. Efficacy of an m-Health Physical Activity and Sleep Health Intervention for Adults: A Randomized Waitlist-Controlled Trial. Am J Prev Med 2019;57:50314. doi:10.1016/j.amepre.2019.05.009

115 Nakata Y, Sasai H, Tsujimoto T, Hashimoto K, Kobayashi H. Webbased intervention to promote weight-loss maintenance using an activity monitor: A randomized controlled trial. Prev Med Rep 2019;14:100839. doi:10.1016/j.pmedr.2019.100839

116 Obling KH, Overgaard K, Juul L, Maindal HT. Effects of a motivational, individual and locally anchored exercise intervention (MILE) on cardiorespiratory fitness: a community-based randomised controlled trial. BMC Public Health 2019;19:239. doi:10.1186/s12889-019 6556-0 
117 Ormel HL, van der Schoot GGF, Westerink NL, Sluiter WJ, Gietema JA, Walenkamp AME. Self-monitoring physical activity with a smartphone application in cancer patients: a randomized feasibility study (SMART-trial). Support Care Cancer 2018;26:3915-23. doi:10.1007/ s00520-018-4263-5

118 Paschali AA, Goodrick GK, Kalantzi-Azizi A, Papadatou D, Balasubramanyam A. Accelerometer feedback to promote physical activity in adults with type 2 diabetes: a pilot study. Percept Mot Skills 2005:100:61-8. doi:10.2466/pms.100.1.61-68

119 Pears S, Bijker M, Morton K, et al, VBI Programme Team. A randomised controlled trial of three very brief interventions for physical activity in primary care. BMC Public Health 2016;16:1033. doi:10.1186/s12889-016-3684-7

120 Pellegrini CA, Verba SD, Otto AD, Helsel DL, Davis KK, Jakicic JM. The comparison of a technology-based system and an in-person behavioral weight loss intervention. Obesity (Silver Spring) 2012;20:356-63. doi:10.1038/oby.2011.13

121 Petersen CB, Severin M, Hansen AW, Curtis T, Grønbæk M, Tolstrup JS. A population-based randomized controlled trial of the effect of combining a pedometer with an intervention toolkit on physical activity among individuals with low levels of physical activity or fitness. Prev Med 2012;54:125-30. doi:10.1016/j.ypmed.2011.12.012

122 Piette JD, Richardson C, Himle J, et al. A randomized trial of telephonic counseling plus walking for depressed diabetes patients. Med Care 2011;49:641-8. doi:10.1097/MLR.0b013e318215d0c9

123 Pillay ID, Kolbe-Alexander TL, Proper KI, et al Steps that count! A feasibility study of a pedometer-based, health-promotion intervention in an employed, South African population. South Afr Sports Med Assoc 2014;26:15-19. doi:10.7196/SAJSM.500

124 Plow M, Finlayson M, Liu J, Motl RW, Bethoux F, Sattar A. Randomized Controlled Trial of a Telephone-Delivered Physical Activity and Fatigue Self-management Interventions in Adults With Multiple Sclerosis. Arch Phys Med Rehabil 2019;100:2006-14 . . doi:10.1016/j. apmr.2019.04.022

125 Polzien KM, Jakicic JM, Tate DF, Otto AD. The efficacy of a technologybased system in a short-term behavioral weight loss intervention. Obesity (Silver Spring) 2007;15:825-30. doi:10.1038/oby.2007.584

126 Pope ZC, Zeng N, Zhang R, Lee HY, Gao Z. Effectiveness of Combined Smartwatch and Social Media Intervention on Breast Cancer Survivor Health Outcomes: A 10-Week Pilot Randomized Trial. J Clin Med 2018;7:E140. doi:10.3390/jcm7060140

127 Poston L, Briley AL, Barr S, et al. Developing a complex intervention for diet and activity behaviour change in obese pregnant women (the UPBEAT trial); assessment of behavioural change and process evaluation in a pilot randomised controlled trial. BMC Pregnancy Childbirth 2013:13:148 doi:10.1186/1471-2393-13-148

128 Recio-Rodriguez II, Agudo-Conde C, Martin-Cantera C, et al, EVIDENT Investigators. Short-Term Effectiveness of a Mobile Phone App for Increasing Physical Activity and Adherence to the Mediterranean Diet in Primary Care: A Randomized Controlled Trial (EVIDENT II Study). I Med Internet Res 2016;18:e331. doi:10.2196/jmir.6814

129 Roos R, Myezwa H, van Aswegen H, Musenge E. Effects of an education and home-based pedometer walking program on ischemic heart disease risk factors in people infected with HIV: a randomized trial. J Acquir Immune Defic Syndr 2014;67:268-76. doi:10.1097/ QAl.0000000000000299

130 Rosenbaum S, Sherrington C, Tiedemann A. Exercise augmentation compared with usual care for post-traumatic stress disorder: a randomized controlled trial. Acta Psychiatr Scand 2015;131:350-9. doi:10.1111/acps.12371

131 Alshahrani A, Siddiqui A, Khalil S, et al. WhatsApp-based intervention for promoting physical activity among female college students, Saudi Arabia: a randomized controlled trial. East Mediterr Health / 2021;27:782-9. doi:10.26719/emhj.21.012

132 Sharp P, Caperchione C. The effects of a pedometer-based intervention on first-year university students: A randomized control trial. / Am Coll Health 2016:64:630-8 doi:10.1080/07448481.2016.1217538

133 Sheshadri A, Kittiskulnam P, Lazar AA, Johansen KL. A Walking Intervention to Increase Weekly Steps in Dialysis Patients: A Pilot Randomized Controlled Trial. Am J Kidney Dis 2020;75:488-96. doi:10.1053/j.ajkd.2019.07.026

134 Singh B, Spence RR, Sandler CX, Tanner J, Hayes SC. Feasibility and effect of a physical activity counselling session with or without provision of an activity tracker on maintenance of physical activity in women with breast cancer - A randomised controlled trial. J Sci Med Sport 2020;23:283-90. doi:10.1016/j.jsams.2019.09.019

135 Sniehotta FF, Evans EH, Sainsbury K, et al. Behavioural intervention for weight loss maintenance versus standard weight advice in adults with obesity: A randomised controlled trial in the UK (NULevel Trial). PLoS Med 2019:16:e1002793 doi:10.1371/journal.pmed.1002793

136 Spence JC, Burgess J, Rodgers W, Murray T. Effect of pretesting on intentions and behaviour: a pedometer and walking intervention. Psychol Health 2009:24:777-89. doi:10.1080/08870440801989938
137 Staudter M, Dramiga S, Webb L, Hernandez D, Cole R. Effectiveness of pedometer use in motivating active duty and other military healthcare beneficiaries to walk more. US Army Med Dep J 2011;108-19.

138 Thomas JG, Raynor HA, Bond DS, et al. Weight loss in Weight Watchers Online with and without an activity tracking device compared to control: A randomized trial. Obesity (Silver Spring) 2017;25:1014-21. doi:10.1002/oby.21846

139 Thorndike AN, Mills S, Sonnenberg L, et al. Activity monitor intervention to promote physical activity of physicians-in-training: randomized controlled trial. PLoS One 2014;9:e100251. doi:10.1371/journal.pone.0100251

140 Tudor-Locke C, Bell RC, Myers AM, et al. Controlled outcome evaluation of the First Step Program: a daily physical activity intervention for individuals with type II diabetes. Int J Obes Relat Metab Disord 2004:28:113-9. doi:10.1038/sj.ijo.0802485

$141 \mathrm{Uhm} \mathrm{KE}$, Yoo JS, Chung SH, et al. Effects of exercise intervention in breast cancer patients: is mobile health (mHealth) with pedometer more effective than conventional program using brochure? Breast Cancer Res Treat 2017;161:443-52. doi:10.1007/s10549-0164065-8

142 Unick JL, O'Leary KC, Bond DS, Wing RR. Physical activity enhancement to a behavioral weight loss program for severely obese individuals: A preliminary investigation. ISRN Obes 2012;2012. doi:10.5402/2012/465158

143 Vallance IK, Courneya KS, Plotnikoff RC, Yasui Y, Mackey IR. Randomized controlled trial of the effects of print materials and step pedometers on physical activity and quality of life in breast cancer survivors. / Clin Oncol 2007;25:2352-9. doi:10.1200/ ICO.2006.07.9988

144 Vallance JK, Friedenreich CM, Lavallee CM, et al. Exploring the Feasibility of a Broad-Reach Physical Activity Behavior Change Intervention for Women Receiving Chemotherapy for Breast Cancer: A Randomized Trial. Cancer Epidemiol Biomarkers Prev 2016;25:3918. doi:10.1158/1055-9965.EPI-15-0812

145 Van Hoye K, Wijtzes Al, Lefevre J, De Baere S, Boen F. Year-round effects of a four-week randomized controlled trial using different types of feedback on employees' physical activity. BMC Public Health 2018;18:492. doi:10.1186/s12889-018-5402-0

146 Vandelanotte C, Duncan MJ, Maher CA, et al. The Effectiveness of a Web-Based Computer-Tailored Physical Activity Intervention Using Fitbit Activity Trackers: Randomized Trial. J Med Internet Res 2018:20:e11321. doi:10.2196/11321

147 Wallbank G, Sherrington C, Canning CG, et al. Active women over 50: study protocol for RCT of a low-dose information and support program to promote physical activity behaviour change. BMC Public Health 2019;19:1225. doi:10.1186/s12889-019-7514-6

148 Walsh JC, Corbett T, Hogan M, Duggan J, McNamara A. An mHealth Intervention Using a Smartphone App to Increase Walking Behavior in Young Adults: A Pilot Study. JMIR Mhealth Uhealth 2016;4:e109. doi:10.2196/mhealth.5227

149 Williams J, Stubbs B, Richardson S, et al. 'Walk this way': results from a pilot randomised controlled trial of a health coaching intervention to reduce sedentary behaviour and increase physical activity in people with serious mental illness. BMC Psychiatry 2019;19:287. doi:10.1186/s12888-019-2274-5

150 Omar NO, Ahmad RA, Mohd Shah MS, Aminuddin AA, Chellappan $\mathrm{KC}$. Amelioration of inflammation in young men with cardiovascular risks participating pedometer-based walking programme. Med J Malaysia 2021:76:375-81.

151 Poirier J, Bennett WL, Jerome GJ, et al. Effectiveness of an Activity Tracker- and Internet-Based Adaptive Walking Program for Adults: A Randomized Controlled Trial. / Med Internet Res 2016;18:e34. doi:10.2196/jmir.5295

152 Anderson AS, Chong HY, Craigie AM, et al. A novel approach to increasing community capacity for weight management a volunteer-delivered programme (ActWELL) initiated within breast screening clinics: a randomised controlled trial. Int J Behav Nutr Phys Act 2021;18:34. doi:10.1186/s12966021-01099-7

153 Li LC, Feehan LM, Xie H, et al. Efficacy of a Physical Activity Counseling Program With Use of a Wearable Tracker in People With Inflammatory Arthritis: A Randomized Controlled Trial. Arthritis Care Res (Hoboken) 2020;72:1755-65. doi:10.1002/acr.24199

154 Kong S, Lee JK, Kang D, et al. Comparing the Effectiveness of a Wearable Activity Tracker in Addition to Counseling and Counseling Only to Reinforce Leisure-Time Physical Activity among Breast Cancer Patients: A Randomized Controlled Trial. Cancers (Basel) 2021;13:2692. doi:10.3390/cancers13112692

155 Haufe S, Kahl KG, Kerling A, et al. Employers With Metabolic Syndrome and Increased Depression/Anxiety Severity Profit Most From Structured Exercise Intervention for Work Ability and Quality of Life. Front Psychiatry 2020;11:562. doi:10.3389/ fpsyt.2020.00562 
156 Freitas PD, Passos NFP, Carvalho-Pinto RM, et al. A Behavior Change Intervention Aimed at Increasing Physical Activity Improves Clinical Control in Adults With Asthma: A Randomized Controlled Trial. Chest 2021;159:46-57. doi:10.1016/j.chest.2020.08.2113

157 Rayward AT, Murawski B, Duncan MJ, et al. Efficacy of an m-Health Physical Activity and Sleep Intervention to Improve Sleep Quality in Middle-Aged Adults: The Refresh Study Randomized Controlled Trial. Ann Behav Med 2020;54:470-83. doi:10.1093/abm/kaz064

158 Hajna S, Sharp SJ, Cooper AJM, et al. Effectiveness of Minimal Contact Interventions: An RCT. Am J Prev Med 2021;60:e111-21. doi:10.1016/j.amepre.2020.10.010

159 Farnell G, Barkley J. The effect of a wearable physical activity monito (Fitbit One) on physical activity behaviour in women: A pilot study. J Hum Sport Exerc 2017;12. doi:10.14198/jhse.2017.124.09

160 Kim H, Kang M. A Tailored Domain-specific Intervention Using Contextual Information about Sedentary Behavior to Reduce Sedentary Time: A Bayesian Approach. Meas Phys Educ Exerc Sci 2021;25:171-9. doi:10.1080/1091367X.2020.1862123

161 Lang AE, Hendrick PA, Clay L, et al. A randomized controlled trial investigating effects of an individualized pedometer driven walking program on chronic low back pain. BMC Musculoskelet Disord 2021:22:206. doi:10.1186/s12891-021-04060-8

162 Yang Y-P, Wang C-J, Wang J-J, et al. The Effects of an Activity Promotion System on active living in overweight subjects with metabolic abnormalities. Obes Res Clin Pract 2017;11:718-27. doi:10.1016/j. orcp.2017.06.002

163 Peacock OJ, Western MJ, Batterham AM, et al. Effect of novel technology-enabled multidimensional physical activity feedback in primary care patients at risk of chronic disease - the MIPACT study: a randomised controlled trial. Int J Behav Nutr Phys Act 2020;17:99. doi:10.1186/s12966-020-00998-5

164 Ryan JM, Fortune J, Stennett A, et al. Safety, feasibility, acceptability and effects of a behaviour-change intervention to change physica activity behaviour among people with multiple sclerosis: Results from the iStep-MS randomised controlled trial. Mult Scler 2020;26:190718. doi:10.1177/1352458519886231

165 Singh B, Spence RR, Sandler CX, Tanner J, Hayes SC. Feasibility and effect of a physical activity counselling session with or without provision of an activity tracker on maintenance of physical activity in women with breast cancer - A randomised controlled trial. J Sci Med Sport 2020;23:283-90. doi:10.1016/j.jsams.2019.09.019

166 Taylor A, Taylor RS, Ingram W, et al. Randomised controlled trial of an augmented exercise referral scheme using web-based behavioural support for inactive adults with chronic health conditions: the e-coachER trial. Br J Sports Med 2021:55:444-50.

167 Nasseri NN, Ghezelbash E, Zhai Y, et al. Feasibility of a smartphone app to enhance physical activity in progressive MS: a pilot randomized controlled pilot trial over three months. Peer/ 2020;8:e9303. doi:10.7717/peeri.9303

168 Teychenne M, Abbott G, Stephens LD, et al. Mums on the Move: A pilot randomised controlled trial of a home-based physical activity intervention for mothers at risk of postnatal depression. Midwifery 2021;93:102898. doi:10.1016/j.midw.2020.102898

169 Sandborg J, Söderström E, Henriksson P, et al. Effectiveness of a Smartphone App to Promote Healthy Weight Gain, Diet, and Physical Activity During Pregnancy (HealthyMoms): Randomized Controlled Trial. JMIR Mhealth Uhealth 2021;9:e26091. doi:10.2196/26091
170 Herman HG, Kleiner I, Tairy D, et al. 623: Improving post-Cesarean mobility with personalized feedback using digital step counters - a randomized controlled trial. Am J Obstet Gynecol 2020;222:S397-8. doi:10.1016/j.ajog.2019.11.639

171 Levin ME, Krafft J, Seifert S, Lillis J. Tracking Valued and Avoidant Functions with Health Behaviors: A Randomized Controlled Trial of the Acceptance and Commitment Therapy Matrix Mobile App. Behav Modif 2022;46:63-89. doi:10.1177/0145445520913987

172 Carr LJ, Karvinen K, Peavler M, Smith R, Cangelosi K. Multicomponent intervention to reduce daily sedentary time: a randomised controlled trial. BMJ Open 2013;3:e003261. doi:10.1136/ bmjopen-2013-003261

173 Hodkinson A, Kontopantelis E, Adeniji C, et al. Accelerometer- and Pedometer-Based Physical Activity Interventions Among Adults With Cardiometabolic Conditions: A Systematic Review and Metaanalysis. JAMA Netw Open 2019;2:e1912895. doi:10.1001/ jamanetworkopen.2019.12895

174 Hall KS, Hyde ET, Bassett DR, et al. Systematic review of the prospective association of daily step counts with risk of mortality, cardiovascular disease, and dysglycemia. Int J Behav Nutr Phys Act 2020;17:78. doi:10.1186/s12966-020-00978-9

175 Hansen BH, Dalene KE, Ekelund U, et al. Step by step: Association of device-measured daily steps with all-cause mortality-A prospective cohort Study. Scand J Med Sci Sports 2020;30:1705-11. doi:10.1111/sms.13726

176 Saint-Maurice PF, Troiano RP, Bassett DRJr, et al. Association of Daily Step Count and Step Intensity With Mortality Among US Adults. JAMA 2020;323:1151-60. doi:10.1001/jama.2020.1382

177 Ekelund U, Tarp J, Steene-Johannessen J, et al. Dose-response associations between accelerometry measured physical activity and sedentary time and all cause mortality: systematic review and harmonised meta-analysis. BMJ 2019;366:14570. doi:10.1136/bmj. 14570

178 Rees-Punia E, Evans EM, Schmidt MD, et al. Mortality Risk Reductions for Replacing Sedentary Time With Physical Activities. Am J Prev Med 2019;56:736-41. doi:10.1016/i.amepre.2018.12.006

179 Mansoubi M, Pearson N, Biddle SJH, Clemes S. The relationship between sedentary behaviour and physical activity in adults: a systematic review. Prev Med 2014;69:28-35. doi:10.1016/j. ypmed.2014.08.028

180 Lee PH, Macfarlane DJ, Lam TH, Stewart SM. Validity of the International Physical Activity Questionnaire Short Form (IPAQSF): a systematic review. Int / Behav Nutr Phys Act 2011;8:115. doi:10.1186/1479-5868-8-115

181 Kanstrup AM, Bertelsen P, Jensen MB. Contradictions in digital health engagement: An activity tracker's ambiguous influence on vulnerable young adults' engagement in own health. Digit Health 2018;4:2055207618775192. doi:10.1177/2055207618775192

182 Copas J. What works?: selectivity models and meta-analysis. / $R$ Stat Soc Ser A Stat Soc 1999:162:95-109. doi:10.1111/1467 985X.00123

183 Copas JB, Shi JQ. A sensitivity analysis for publication bias in systematic reviews. Stat Methods Med Res 2001:10:251-65. doi:10.1177/096228020101000402

Web appendix: Appendix 1-3 